\title{
New Synthetic Routes for 1-Benzyl-1,4,7,10-tetraazacyclododecane and 1,4,7,10-Tetraazacyclododecane-1-acetic Acid Ethyl Ester, Important Starting Materials for Metal-coded DOTA-Based Affinity Tags
}

\author{
Stephan W. Kohl ${ }^{\mathrm{a}}$, Katharina Kuse ${ }^{\mathrm{a}}$, Markus Hummert ${ }^{\mathrm{a}}$, Herbert Schumann ${ }^{\mathrm{a}}$, \\ Clemens Mügge ${ }^{\mathrm{b}}$, Katharina Janek ${ }^{\mathrm{c}}$, and Hardy Weißhoff ${ }^{\mathrm{b}}$ \\ ${ }^{a}$ Institut für Chemie, Technische Universität Berlin, Straße des 17. Juni 135, D-10623 Berlin, \\ Germany \\ b Institut für Chemie, Humboldt-Universität zu Berlin, Brook-Taylor-Straße 2, D-12489 Berlin, \\ Germany \\ c Universitätsklinikum Charité, Humboldt-Universität zu Berlin, Monbijoustraße 2, D-10098 Berlin, \\ Germany
}

Reprint requests to Prof. Dr. H. Schumann. Fax +493031422168.

E-mail: schumann@chem.tu-berlin.de

Z. Naturforsch. 2007, 62b, 397 -406; received November 7, 2006

Dedicated to Prof. Helgard G. Raubenheimer on the occasion of his $65^{\text {th }}$ birthday

\begin{abstract}
Two improved routes to synthesize 1-benzyl-1,4,7,10-tetraazacyclododecane (6) and 1,4,7,10tetraazacyclododecane-1-acetic acid ethyl ester (11) are described as well as the synthesis of 1-\{2-[4-(maleimido-N-propylacetamidobutyl)amino]-2-oxoethyl $\}-1,4,7,10$-tetraazacyclododecane-4,7,10-triacetic acid (17) and its Y, Ho, Tm, and Lu complexes. The ${ }^{1} \mathrm{H}$ and ${ }^{13} \mathrm{C}$ NMR spectra of the new compounds as well as the single crystal X-ray structure analyses of the intermediates 4-benzyl-1,7-bis( $p$-toluenesulfonyl)diethylenetriamine (3) and 1,4,7-tris ( $p$-toluenesulfonyl)diethylenetriamine (7) are reported and discussed. The rare earth complexes of $\mathbf{1 7}$ have been characterized by ${ }^{1} \mathrm{H}$ NMR spectroscopy and MALDI-TOF mass spectrometry.
\end{abstract}

Key words: Tetraazacyclododecane, Macrocycle, DOTA, Affinity Tag, Rare Earth Complexes

\section{Introduction}

Macrocyclic polyaminopolycarboxylates have been intensively studied because of their numerous applications, which often require selective functionalization [1,2]. Metal ion-conjugated peptides with 1,4, 7,10-tetraazacyclododecane-1,4,7,10-tetraacetic acid (DOTA), 1,4,7-triazacyclononane-1,4,7-triacetic acid (NOTA) or 1,4,8,11-tetraazacyclotetradecane-1,4,8, 11-tetraacetic acid (TETA) ligands are ideal agents for a spectrum of applications in biomedicine, as therapeutic radiopharmaceuticals, luminescent probes for biochemical analysis, or MRI contrast agents [3-6]. Recently a new class of DOTA conjugates was introduced, the so-called element- or metal-coded affinity tags (MECAT) [7,8]. These reagents can be used in quantitative proteomics, as an additional or alternative method to established 2D-GE and recently developed methods employing isotope-coded affinity tags (ICAT) and isobaric tags for relative and absolute quantitation (ITRAQ) [9-13].

Metal-coded affinity tags are reagents composed of a chelating ligand, a monoisotopic metal ion, predominantly rare earth cations, and a reactive group with specificity towards thiol or amino groups. The affinity can be achieved for example by an incorporated group like biotin [11] or by interaction with antibodies [4, 14]. The principle of MECATs is derived from ICAT, but instead of the stable isotope labeling of proteins or peptides a metal ion labeling is applied. The protein mixture of two or more sets of cell states is independently labeled with MECAT reagents containing different metal ions; the samples are combined, and then conventionally cleaved. The MECAT labeled peptides are isolated by affinity chromatography and analyzed by LC-ESI-MS/MS. Peptide sequence information is obtained by tandem mass spectrometry and computer searches of protein data banks. Quantitation of proteins in two cell 


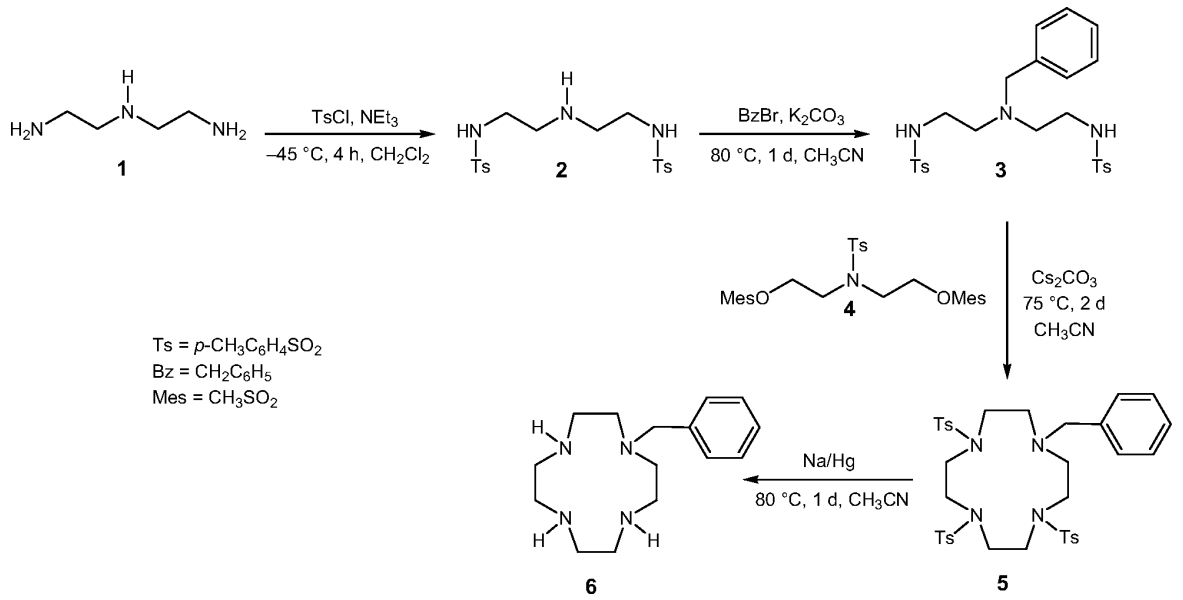

Scheme 1. Route I for the synthesis of 1-benzyl1,4,7,10-tetraazacyclododecane (6). states is performed by comparing the intensity of the identical peptide peak pair from the samples defined by the mass difference of the complex ions chosen.

Metal ions, and particularly rare earth cations, are suitable for ICP-MS and permit low detection limits of quantitation. Many of the rare earth elements are naturally monoisotopic. Thus, a variety of MECATs with desired mass differences can be synthesized by pairwise integration into ligands. Considering only seven monoisotopic rare earth elements, 19 different mass tags are produceable with mass differences from $2 \mathrm{Da}$ for ${ }^{139} \mathrm{La} /{ }^{141} \mathrm{Pr}$ to $86 \mathrm{Da}$ for ${ }^{89} \mathrm{Y} /{ }^{175} \mathrm{Lu}$. Thereby more than two samples can be investigated in parallel, or ambiguous analytical results can be verified in an independent run.

For about 15 years, several research groups have been engaged in the synthesis of mono-functionalized DOTA derivatives [15-18]. Meanwhile, $\mathrm{N}-$ and C-functionalized DOTA derivatives are commercially available, but still very expensive. To make these important compounds more readily available, we describe in this paper two suitable, cost-efficient synthetic routes to 1-benzyl-1,4,7,10-tetraazacyclododecane (6) [19], 1,4,7,10-tetraazacyclododecane-1-acetic acid ethyl ester (11) [20] and 1,4,7,10-tetraazacyclododecane-1-acetic acid-4,7,10-tris-(acetic acid tertbutyl ester) (tris- $\left.{ }^{t} \mathrm{Bu}-\mathrm{DOTA}\right)$ (15) [17], the starting materials for the synthesis of $\mathrm{N}$-functionalized DOTA ligands, as well as the synthesis of 1\{2-[4-(maleimido-N-propylacetamidobutyl)amino]-2oxoethyl $\}$-1,4,7,10-tetraazacyclododecane-4,7,10-triacetic acid (17) and its Y, Ho, Tm, and Lu complexes.

\section{Results and Discussion}

Synthesis of 1-benzyl-1,4,7,10-tetraazacyclododecane (6) and 1,4,7,10-tetraazacyclododecane-1-acetic acid ethyl ester (11)

$\mathrm{N}$-substituted tetraazacyclododecanes are generally synthesized starting with diethylenetriamine (1) and diethanolamine as common educts via bimolecular cyclization reactions using toluenesulfonyl protecting groups, with subsequent deprotection. To improve our recently published procedure [21], we used a modified way (Scheme 1). The first step, the selective tosylation of the two primary amino groups of $\mathbf{1}$ is possible at $-45{ }^{\circ} \mathrm{C}$ in dichloromethane. Thus, the protection and deprotection of the terminal amino groups with phthalic anhydride can be avoided and the yield of 1,7bis ( $p$-toluenesulfonyl)diethylenetriamine (2) [22] is increased. The tri-tosylated by-product, 1,4,7-tris $(p$ toluenesulfonyl)diethylenetriamine (7) [23], can easily be separated by filtration and used for further preparations. Alkylation of $\mathbf{2}$ with benzyl bromide and an excess of $\mathrm{K}_{2} \mathrm{CO}_{3}$ results in the formation of 4-benzyl1,7-bis ( $p$-toluenesulfonyl)diethylenetriamine (3) [21], which crystallizes after a few weeks as colorless crystals. Cyclization with 1,5-bis(methylsulfonyloxy)3-aza-3-( $p$-toluenesulfonylamido)pentane (4) [24] according to [21] and elimination of the protecting groups by sodium amalgam yields the monosubstituted cyclen 6 [19] in $80 \%$ yield.

Route II for the synthesis of $\mathbf{6}$ starts with the complete tosylation of $\mathbf{1}$ at $0{ }^{\circ} \mathrm{C}$ yielding $\mathbf{7}$ [23] as a white powder which forms colorless single crystals from acetone suitable for X-ray analysis (Scheme 2). Cyclization of 7 with the bis-methylsulf- 


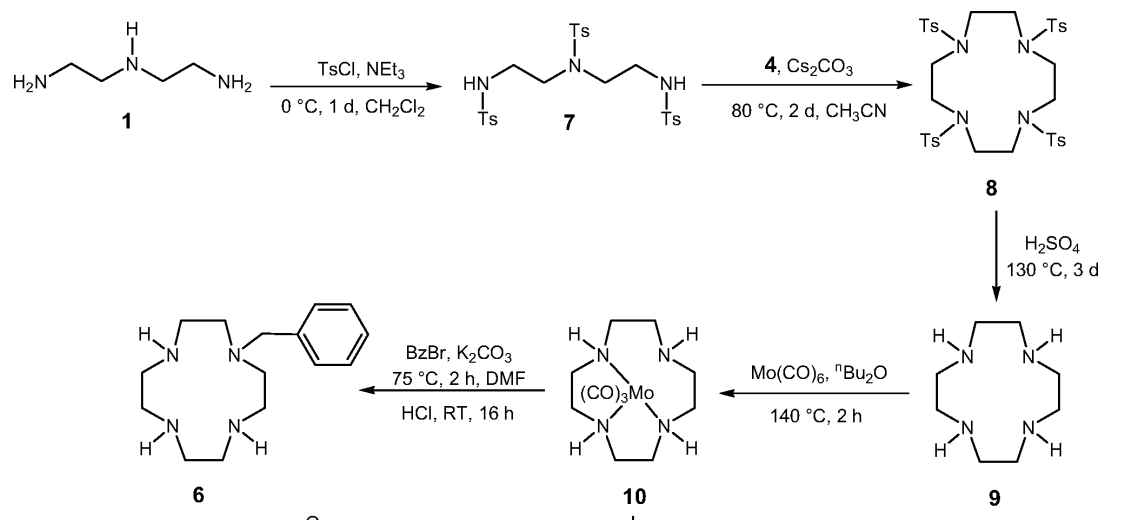<smiles>CCOC(=O)CN1CCNCCNCCN(C)CCN1</smiles>

onyloxy compound 4 yields 1,4,7,10-tetrakis ( $p$-toluenesulfonyl)-1,4,7,10-tetraazacyclododecane (8) [25], which is converted into 1,4,7,10-tetraazacyclododecane (cyclen) (9) by heating in concentrated $\mathrm{H}_{2} \mathrm{SO}_{4}$ for three days [26]. Reaction with $\mathrm{Mo}(\mathrm{CO})_{6}$ following the procedure described by Patinec et al. [27, 28] resulted in $\eta^{3}-1,4,7,10$-tetraazacyclododecane molybdenumtricarbonyl (10) [26], which was alkylated with benzylbromide and bromoacetic acid ethyl ester in DMF followed by decoordination from the $\mathrm{Mo}(\mathrm{CO})_{3}$ fragment by $\mathrm{HCl}$ yielding 6 and 1,4,7,10-tetraazacyclododecane-1-acetic acid ethyl ester (11) [20], respectively.

Molecular structure of 4-benzyl-1,7-bis(p-toluenesulfonyl)diethylenetriamine (3) and 1,4,7-tris(p-toluenesulfonyl)diethylenetriamine (7)

The structure of monoclinic crystals of the ditosylated benzylated triamine 3 (Fig. 1) shows nonexceptional averaged bond lengths $\mathrm{C}-\mathrm{N}(1.47 \AA)$ and $\mathrm{C}-\mathrm{C}(1.50 \AA)$ along the chain of the triamine. Similar distances $\mathrm{C}-\mathrm{N}(1.46 \AA)$ and $\mathrm{C}-\mathrm{C}(1.52 \AA)$ were found in the structure of the monoclinic crystals of the tritosylated diethylenetriamine 7 (Fig. 2). The mean $\mathrm{N}-\mathrm{S}$ distances in the structures of $\mathbf{3}$ and $\mathbf{7}$ are 1.61 and $1.62 \AA$, respectively. The conformation of the dimethylene units in the amine chain is $\mathrm{N}(1)-\mathrm{C}(8)-\mathrm{C}(9)-$ $\mathrm{N}(2) 66^{\circ}((+)$-synclinal $)$ and $\mathrm{N}(1)-\mathrm{C}(10)-\mathrm{C}(11)-\mathrm{N}(3)$ $-58^{\circ}((-)$-synclinal) for compound 3 and $\mathrm{N}(2)-\mathrm{C}(3)-$ $\mathrm{C}(4)-\mathrm{N}(3) 179^{\circ}$ (antiperiplanar) and $\mathrm{N}(1)-\mathrm{C}(1)$ $\mathrm{C}(2)-\mathrm{N}(2) 69^{\circ}((+)-$ synclinal $)$ for compound 7 . For 3

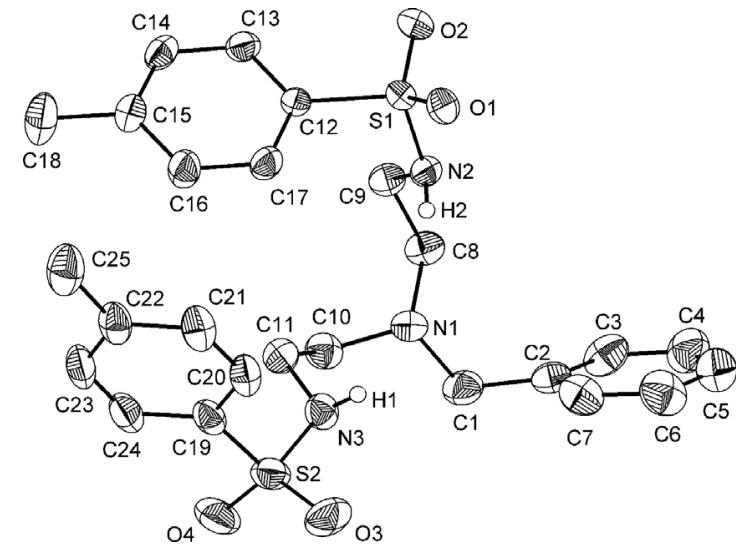

Fig. 1. ORTEP [29] presentation of the molecular structure of 3 (displacement ellipsoids at the $30 \%$ probability level); all hydrogen atoms except $\mathrm{H}(1)$ and $\mathrm{H}(2)$ have been omitted for clarity; selected bond lengths $(\AA)$ and angles (deg): $\mathrm{N}(1)-\mathrm{C}(1) \quad 1.476(3), \quad \mathrm{N}(1)-\mathrm{C}(8)$ 1.460(3), $\mathrm{N}(1)-\mathrm{C}(10) \quad 1.476(3), \quad \mathrm{N}(2)-\mathrm{C}(9) \quad 1.472(3), \quad \mathrm{N}(2)-\mathrm{S}(1)$

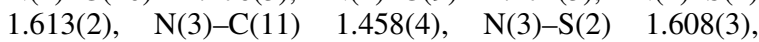
$\mathrm{N}(2)-\mathrm{O}(1)$ 3.165(3), N(3)-O(3) 3.090(3); C(9)-N(2)-S(1) 118.11(17), C(11)-N(3)-S(2) 120.4(2).

the distances between the nitrogene atoms $\mathrm{N}(2)$ and $\mathrm{N}(3)$ and the oxygen atom $\mathrm{O}(1)$ of the adjacent molecule are 3.17 and $3.09 \AA$, respectively. The two molecules are linked via intermolecular hydrogen bonds. The bonding of two nitrogen atoms to only one oxygen atom is the reason for the close proximity (3-4 $\AA$ ) of the two tosyl groups in this molecule, compared with 7, where the terminal tosyl-groups are located far away from each other $(9-10 \AA)$. Intermolec- 


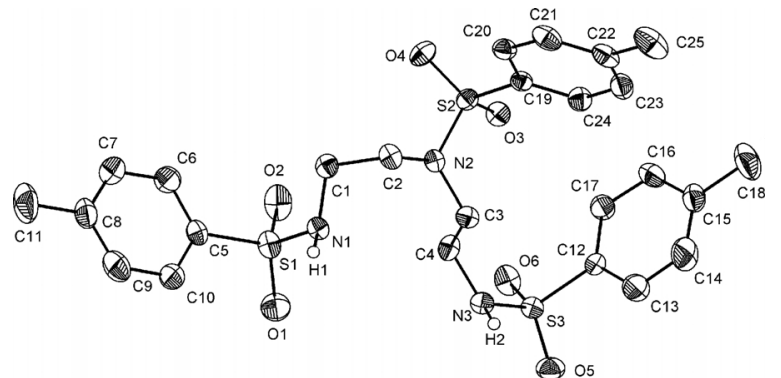

Fig. 2. ORTEP [29] presentation of the molecular structure of 7 (30\% probability ellipsoids); all hydrogen atoms except $\mathrm{H}(1)$ and $\mathrm{H}(2)$ have been omitted for clarity; selected bond lengths (A): $\mathrm{N}(1)-\mathrm{C}(1)$ 1.462(5), N(1)-S(1) 1.620(3), $\mathrm{N}(2)-\mathrm{C}(2) \quad 1.473(4), \quad \mathrm{N}(2)-\mathrm{C}(3) \quad 1.467(4), \quad \mathrm{N}(2)-\mathrm{S}(2)$ 1.473(4), $\mathrm{N}(3)-\mathrm{C}(4) \quad 1.526(5), \quad \mathrm{N}(3)-\mathrm{S}(3) \quad 1.610(3)$, $\mathrm{N}(1)-\mathrm{O}(2) 3.008(4), \mathrm{N}(3)-\mathrm{O}(3) 2.923(4)$.

ular hydrogen bonds located in 7 between $\mathrm{N}(1)$ and $\mathrm{O}(2)(3.01 \AA)$ and between $\mathrm{N}(3)$ and $\mathrm{O}(6)(2.92 \AA)$ lead to a network in the crystal.

Synthesis of 1-\{2-[4-(maleimido- $N$-propylacetamidobutyl)amino]-2-oxoethyl\}-1,4,7,10-tetraazacyclododecane-4,7,10-triacetic acid (17) and its Y, Ho, Tm, and Lu complexes

Compounds $\mathbf{6}$ and $\mathbf{1 1}$ are the key compounds for the synthesis of 1,4,7,10-tetraazacyclododecane-4,7,10-tris(acetic acid tert-butyl ester)-1-acetic acid (tris- ${ }^{t} \mathrm{Bu}-\mathrm{DOTA}$ ) (15) [17], which in turn is the starting material for the synthesis of $\mathrm{N}$-functionalized DOTA ligands, which are commercially available, but very expensive. Following published routes [16, 30, 31], 15 is prepared either starting from $\mathbf{6}$ by alkylation of the unprotected amine functions with $\mathrm{BrCH}_{2} \mathrm{COO}^{t} \mathrm{Bu}$ to yield 1,4,7,10-tetraazacyclododecane-1-benzyl-4,7,10-tris(acetic acid tert-butyl ester) (12), followed by removal of the protecting benzyl group with $\mathrm{H}_{2} / \mathrm{Pd} / \mathrm{C}$ to produce $1,4,7,10$-tetraazacyclododecane-4,7,10-tris(acetic acid tert-butyl ester) (13) [32] and finally by incorporation of an acetate group or from 11 in two steps via 1,4,7,10-tetraazacyclododecane-1-acetic acid ethyl ester-4,7,10-tris (acetic acid tert-butyl ester) (14) (Scheme 3).

The triply protected DOTA-derivative 15 reacts with 2-(1H-7-azabenzotriazol-1-yl)-1,1,3,3-tetramethyluronium hexafluorophosphate (HATU), Hünig's base (DIEA) and 1,4-diaminobutane trityl resin in DMF with formation of the resin-fixed tris-tert-butyl ester of 2-(1,4,7,10-tetraaza-4,7,10-tris(carboxymethyl)-1-cyclododecyl)-acetyl-diaminobutane, which is

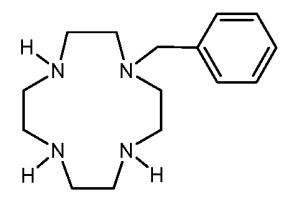

6

$\mathrm{BrCH}_{2} \mathrm{CO}_{2}{ }^{\mathrm{t}} \mathrm{Bu}$ $\mathrm{K}_{2} \mathrm{CO}_{3}$, DMF $80^{\circ} \mathrm{C}, 20 \mathrm{~h}$

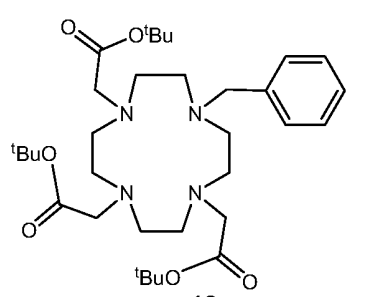

12

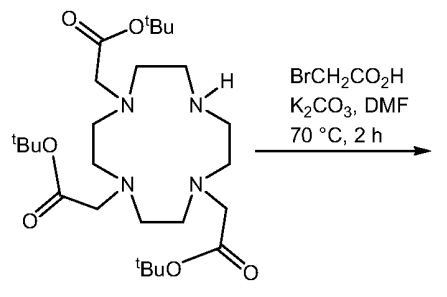

13

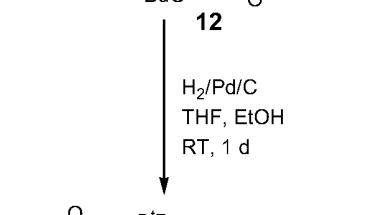<smiles>CCOC(=O)CN1CCNCCNCCN1</smiles>

11 $\mathrm{BrCH}_{2} \mathrm{CO}_{2}{ }^{\mathrm{t}} \mathrm{Bu}$ $\mathrm{K}_{2} \mathrm{CO}_{3}$, DMF $80^{\circ} \mathrm{C}, 20 \mathrm{~h}$

$\checkmark$<smiles>CCCCCN1CCN(CC(=O)OCC)CCN(CC(=O)OCC)CCN(CC(=O)OCC)CC1</smiles><smiles>CCCCOC(=O)CN1CCN(CC(=O)OCCC)CCN(CC(=O)OCCC)CCN(CC(=O)OCCC)CC1</smiles>

15
Scheme 3. Synthesis of tris- ${ }^{t}$ Bu-DOTA (15).

deprotected and cleaved from the resin by trifluoroacetic acid (TFA), water and triisopropylsilane (TPS) yielding 16 in $84 \%$ yield as a white solid (Scheme 4 ).

Compound 16 reacts with $\beta$-maleimidopropionic acid $N$-hydroxysuccinimid ester in the presence of triethylamine in DMF yielding 1-\{2-[4-(maleimido-N-propylacetamidobutyl)amino]-2-oxoethyl $\}$ 1,4,7,10-tetraazacyclododecane-4,7,10-triacetic acid (17) in $73 \%$ yield as a white solid. Its reaction with lanthanide trichlorides in water at $\mathrm{pH} 7.0$ results in the formation of lanthanide(III) complexes. The yttrium, holmium, thulium, and lutetium complexes 18, 19, 20, and 21 have been characterized by MALDI-TOF mass spectrometry. Fig. 3 shows the MALDI-TOF mass spectrum of $\mathbf{1 7}$ chelating $\mathrm{Y}^{3+}, \mathrm{Ho}^{3+}, \mathrm{Tm}^{3+}$ and $\mathrm{Lu}^{3+}$ ions. The spectrum demonstrates the utility of rare earths embedded in peptide specific labels as internal standards for quantitative proteomics based 


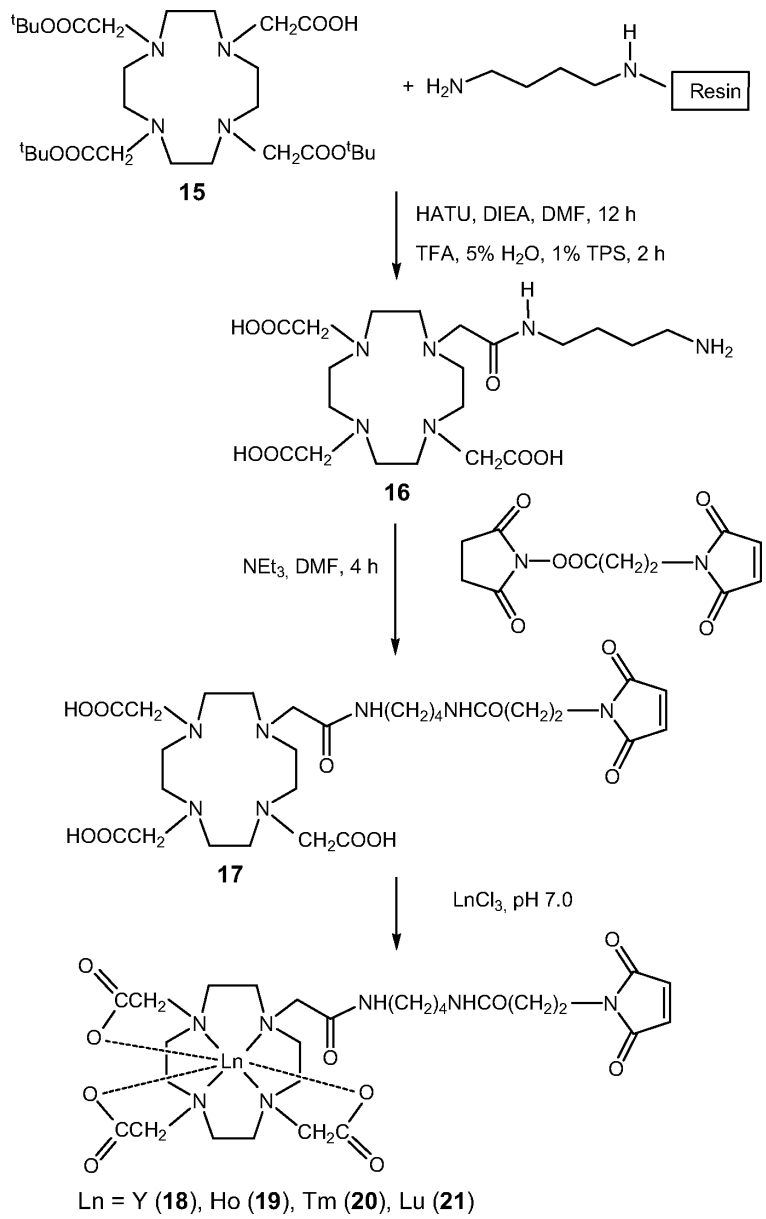

Scheme 4. Synthesis of 1-\{2-[4-(maleimido-N-propylacetamidobutyl)amino]-2-oxoethyl $\}$-1,4,7,10-tetraazacyclododedecane-4,7,10-triacetic acid (17) and the lanthanide complexes 18-21.

on mass spectrometry. Here, mass tags with differences from $4 \mathrm{Da}$ for ${ }^{165} \mathrm{Ho} /{ }^{169} \mathrm{Tm}-17$ to $86 \mathrm{Da}$ for ${ }^{89} \mathrm{Y} /{ }^{175} \mathrm{Lu}-\mathbf{1 7}$ are shown as an example for the variety of different combinations. The use of rare earth elements in addition has the advantage that quantitation can be accomplished by means of ICP MS with very high efficiency and sensitivity. Furthermore, the mass differences between the heavy rare earth-containing tags is useful for the peptide and protein identification in complex mixtures [33].

The lutetium complex 21 was also characterized by elemental analysis and ${ }^{1} \mathrm{H}$ and ${ }^{13} \mathrm{C}$ NMR spectroscopy. These spectra as well as those of $\mathbf{1 6}$ and $\mathbf{1 7}$ are complicated and very hard to assign because of internal hydrogen bonds which cause very broad signals for the macrocyclic $\mathrm{CH}_{2}$ protons at low $\mathrm{pH}$ values [20,34].

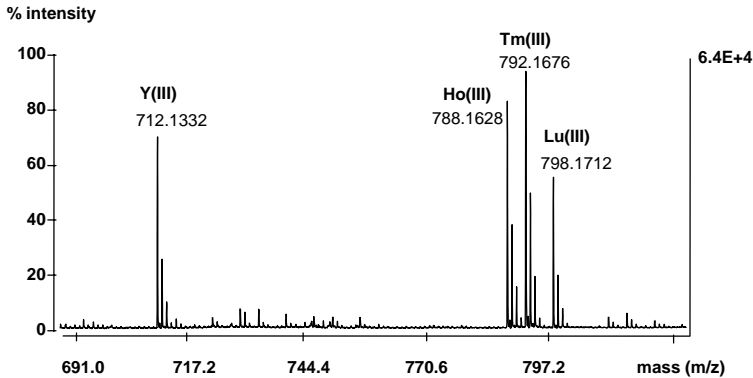

Fig. 3. MALDI-TOF Mass spectrum of the potential thiolspecific MECAT ligand 17 chelating Y, Ho, Tm, and Lu ions.

Further investigations concerning structural analysis of the lanthanide complexes and their application are in progress.

\section{Experimental Section}

Unless noted otherwise, all reactions were carried out at r.t. in dried solvents under dry dinitrogen, using standard Schlenk techniques. Chemicals were purchased from Aldrich, Acros, Chempur, and Macrocyclics and used without further purification. $p$ - $\mathrm{CH}_{3} \mathrm{C}_{6} \mathrm{H}_{4} \mathrm{SO}_{2} \mathrm{~N}\left(\mathrm{CH}_{2} \mathrm{CH}_{2} \mathrm{OSO}_{2}\right.$ $\left.\mathrm{CH}_{3}\right)_{2}$ (4) was prepared according to the literature [24]. ${ }^{1} \mathrm{H}$ and ${ }^{13} \mathrm{C}$ NMR spectra were recorded with Bruker ARX 200 and Bruker AV 400 spectrometers. Chemical shifts $\delta$ were references to TMS or 3-(trimethylsilyl)-propionic acid-D sodium salt (TSP) for measurements in $\mathrm{D}_{2} \mathrm{O}$. Signs of coupling constants were not determined. The MALDI-TOF spectra were recorded with a MALDI-TOF/TOF 4700 Proteomics Analyzer (Applied Biosystems, Framingham, MA, USA). Elemental analyses were carried out using a Thermo Finnigan, Flash EA, 1112 Series analyzer.

$\mathrm{HN}\left(\mathrm{CH}_{2} \mathrm{CH}_{2} \mathrm{NHSO}_{2} \mathrm{C}_{6} \mathrm{H}_{4} \mathrm{CH}_{3}-\mathrm{p}\right)_{2}$ (2). Diethylenetriamine $(10.0 \mathrm{~g}, 0.097 \mathrm{~mol})$ and triethylamine $(19.2 \mathrm{~g}$, $0.190 \mathrm{~mol})$ were dissolved in $\mathrm{CH}_{2} \mathrm{Cl}_{2}(300 \mathrm{~mL})$ and cooled to $-48{ }^{\circ} \mathrm{C}$. To this solution $p$-toluenesulfonylchloride $(36.2 \mathrm{~g}, 0.190 \mathrm{~mol})$ in $\mathrm{CH}_{2} \mathrm{Cl}_{2}(100 \mathrm{~mL})$ was added over a period of $4 \mathrm{~h}$. The temperature did not exceed $-45^{\circ} \mathrm{C}$. After that, the mixture was stirred for $4 \mathrm{~h}$ at r.t., and washed three times with water. The organic layer was dried with $\mathrm{Na}_{2} \mathrm{SO}_{4}$ and the solvents evaporated to give a colorless oil, which was further dried in vacuum. The residue was crystallized from $\mathrm{CH}_{2} \mathrm{Cl}_{2} / \mathrm{CH}_{3} \mathrm{OH}(1: 3)$. Beside the favored ditosylated oily product (2), the crystalline tri-tosylated product 7 is formed (m. p. $59-61{ }^{\circ} \mathrm{C}$ ). Yield: $30.0 \mathrm{~g}(75 \%)$ for $\mathbf{2}$ and $6.0 \mathrm{~g}(10 \%)$ for $7 .-{ }^{1} \mathrm{H} \mathrm{NMR}\left(25^{\circ} \mathrm{C}, 200 \mathrm{MHz}, \mathrm{CDCl}_{3}\right)$ : $\delta=2.35\left(\mathrm{~s}, 6 \mathrm{H}, \mathrm{Ts}-\mathrm{CH}_{3}\right), 2.51\left(\mathrm{~m}, 4 \mathrm{H}\right.$, Ts- $\left.\mathrm{NHCH}_{2} \mathrm{CH}_{2} \mathrm{~N}\right)$, $2.86\left(\mathrm{~m}, 4 \mathrm{H}\right.$, Ts- $\left.\mathrm{NHCH}_{2} \mathrm{CH}_{2} \mathrm{~N}\right), 4.56$ (br, $\left.3 \mathrm{H}, \mathrm{NH}\right), 7.20$ $\left(\mathrm{m}, 4 \mathrm{H}, \mathrm{SO}_{2} \mathrm{CCHCH}\right), 7.66\left(\mathrm{~m}, 4 \mathrm{H}, \mathrm{SO}_{2} \mathrm{CCHCH}\right)$. ${ }^{13} \mathrm{C}$ NMR $\left(25{ }^{\circ} \mathrm{C}, 100.64 \mathrm{MHz}, \mathrm{CDCl}_{3}\right): \delta=21.0$ (Ts$\left.\mathrm{CH}_{3}\right), 41.9\left(\mathrm{NHCH}_{2} \mathrm{CH}_{2} \mathrm{NH}-\mathrm{Ts}\right), 47.3\left(\mathrm{NHCH}_{2} \mathrm{CH}_{2} \mathrm{NH}-\right.$ 
Ts), $126.6\left(2 \times \mathrm{SO}_{2} \mathrm{CCHCH}\right), 129.3\left(2 \times \mathrm{SO}_{2} \mathrm{CCHCH}\right)$, $136.3\left(2 \times \mathrm{SO}_{2} \mathrm{CCH}\right), \quad 142.9\left(2 \times \mathrm{SO}_{2} \mathrm{CCHCHCCH}_{3}\right)$. $\mathrm{C}_{18} \mathrm{H}_{25} \mathrm{~N}_{3} \mathrm{O}_{4} \mathrm{~S}_{2}$ (411.53): calcd. C 52.53, H 6.12, N 10.21, $\mathrm{S}$ 15.58; found C 52.45, H 5.99, N 10.12, S 15.55.

$\mathrm{C}_{6} \mathrm{H}_{5} \mathrm{CH}_{2} \mathrm{~N}\left(\mathrm{CH}_{2} \mathrm{CH}_{2} \mathrm{NHSO}_{2} \mathrm{C}_{6} \mathrm{H}_{4} \mathrm{CH}_{3}-\mathrm{p}\right)_{2}$ (3). The tosylated trisamine 2 (5.8 g, $14 \mathrm{mmol})$ was dissolved in $\mathrm{CH}_{3}$ $\mathrm{CN}(200 \mathrm{~mL})$ and $5.5 \mathrm{~g}(39 \mathrm{mmol})$ of dried $\mathrm{K}_{2} \mathrm{CO}_{3}$ were added. The mixture was heated to $80{ }^{\circ} \mathrm{C}$ and after rapid dropwise addition of $\mathrm{C}_{6} \mathrm{H}_{5} \mathrm{CH}_{2} \mathrm{Br}(1.7 \mathrm{~g}, 14 \mathrm{mmol})$ refluxed for $24 \mathrm{~h}$. The resulting precipitate was filtered and washed 3 times with $\mathrm{CH}_{2} \mathrm{Cl}_{2}(30 \mathrm{~mL})$. The organic layers were washed with water $(4 \times 40 \mathrm{~mL})$ and dried with $\mathrm{Na}_{2} \mathrm{SO}_{4}$. The solvent was removed by rotary evaporation to leave $\mathbf{3}$ as a pale yellow oil, which crystallizes after several days. M.p. $52{ }^{\circ} \mathrm{C}$. Yield: 6.9 g $(98 \%) .-{ }^{1} \mathrm{H}$ NMR $\left(25{ }^{\circ} \mathrm{C}, 400 \mathrm{MHz}, \mathrm{CDCl}_{3}\right): \delta=2.30\left(\mathrm{~s}, 6 \mathrm{H}, \mathrm{CH}_{3}\right), 2.35-$ $2.40\left(\mathrm{~m}, 4 \mathrm{H}, \mathrm{NCH}_{2} \mathrm{CH}_{2} \mathrm{NH}\right), 2.85$ (br, $4 \mathrm{H}, \mathrm{NCH}_{2} \mathrm{CH}_{2} \mathrm{NH}$ ), 3.50 (s, $2 \mathrm{H}$, benzyl- $\left.\mathrm{CH}_{2}\right), 5.64$ (br; $\left.2 \mathrm{H}, \mathrm{NH}\right), 7.13-7.26$ (m, $5 \mathrm{H}$, benzyl-H), 7.27-7.29 (m, $\left.4 \mathrm{H}, \mathrm{SO}_{2} \mathrm{CCHCH}\right)$, $7.63-7.75\left(\mathrm{~m}, 4 \mathrm{H}, \mathrm{SO}_{2} \mathrm{CCHCH}\right) .-{ }^{13} \mathrm{C} \mathrm{NMR}\left(25^{\circ} \mathrm{C}\right.$, $\left.100.64 \mathrm{MHz}, \mathrm{CDCl}_{3}\right): \delta=21.3\left(\mathrm{Ts}_{-} \mathrm{CH}_{3}\right), 44.9(\mathrm{NH}-$ $\left.\mathrm{CH}_{2} \mathrm{CH}_{2} \mathrm{NH}-\mathrm{Ts}\right), 51.3$ ( $\left.\mathrm{NHCH}_{2} \mathrm{CH}_{2} \mathrm{NH}-\mathrm{Ts}\right), 59.3\left(\mathrm{PhCH}_{2}-\right)$, $126.8\left(2 \times \mathrm{SO}_{2} \mathrm{CCHCH}\right), 127.35\left(\mathrm{CH}_{2} \mathrm{CCHCHCH}\right), 128.34$ $\left(\mathrm{CH}_{2} \mathrm{CCHCH}\right), 129.92\left(\mathrm{CH}_{2} \mathrm{CCHCH}\right), 130.1\left(2 \times \mathrm{SO}_{2} \mathrm{C}-\right.$ $\mathrm{CHCH}), 136.1\left(2 \times \mathrm{SO}_{2} \mathrm{CCH}\right), 136.31\left(\mathrm{CH}_{2} \mathrm{CCH}\right), 142.7$ $\left(2 \times \mathrm{SO}_{2} \mathrm{CCHCHC}\right) .-\mathrm{C}_{25} \mathrm{H}_{31} \mathrm{~N}_{3} \mathrm{O}_{4} \mathrm{~S}_{2}$ (501.66): calcd. C 59.86, H 6.23, N 8.38, S 12.78; found C 59.55, H 6.13, $\mathrm{N} 8.20, \mathrm{~S} 12.71$.

$\mathrm{C}_{6} \mathrm{H}_{5} \mathrm{CH}_{2} \mathrm{~N}\left[\mathrm{CH}_{2} \mathrm{CH}_{2} \mathrm{~N}\left(\mathrm{SO}_{2} \mathrm{C}_{6} \mathrm{H}_{4} \mathrm{CH}_{3}-\mathrm{p}\right)\right]_{3} \mathrm{CH}_{2} \mathrm{CH}_{2}$ (5) was prepared according to [21] from $10.7 \mathrm{~g}(21 \mathrm{mmol})$ of $\mathbf{3}$ and $8.9 \mathrm{~g}(21 \mathrm{mmol})$ of 4 . M. p. $161-164{ }^{\circ} \mathrm{C}$. Yield: $9.9 \mathrm{~g}$ $(65 \%) .-{ }^{1} \mathrm{H}$ NMR $\left(25{ }^{\circ} \mathrm{C}, 400 \mathrm{MHz}, \mathrm{CDCl}_{3}\right): \delta=2.39$ (s, $6 \mathrm{H}$, Ts- $\mathrm{CH}_{3}$ ), 2.44 (s, $\left.3 \mathrm{H}, \mathrm{Ts}-\mathrm{CH}_{3}\right), 2.73$ (dd, $4 \mathrm{H}$, benzyl- $\mathrm{NCH}_{2}$ ), 3.08 (dd, $4 \mathrm{H}$, benzyl- $\mathrm{NCH}_{2} \mathrm{CH}_{2}$ ), 3.34 (dd, $4 \mathrm{H}$, benzyl- $\mathrm{NCH}_{2} \mathrm{CH}_{2} \mathrm{NCH}_{2}$ ), 3.46 (dd, $4 \mathrm{H}$, benzyl- $\mathrm{NCH}_{2} \mathrm{CH}_{2}$ $\mathrm{NCH}_{2} \mathrm{CH}_{2}$ ), 3.61 (s, $\left.2 \mathrm{H}, \mathrm{PhCH}_{2}-\right), 7.14-7.20$ (m, $5 \mathrm{H}, \mathrm{Ph}$ ), $7.26\left(\mathrm{~m}, 4 \mathrm{H}, \mathrm{SO}_{2} \mathrm{CCHCH}\right), 7.33\left(\mathrm{~m}, 2 \mathrm{H}, \mathrm{SO}_{2} \mathrm{CCHCH}\right)$, $7.57\left(\mathrm{~m}, 4 \mathrm{H}, \mathrm{SO}_{2} \mathrm{CCHCH}\right), 7.72\left(\mathrm{~m}, 2 \mathrm{H}, \mathrm{SO}_{2} \mathrm{CCHCH}\right)$. $-{ }^{13} \mathrm{C}$ NMR $\left(25{ }^{\circ} \mathrm{C}, 100.64 \mathrm{MHz}, \mathrm{CDCl}_{3}\right): \delta=21.48$ (Ts- $\left.\mathrm{CH}_{3}\right), 21.53\left(2 \times \mathrm{Ts}^{-} \mathrm{CH}_{3}\right), 48.62$ (benzyl- $\left.\mathrm{NCH}_{2} \mathrm{CH}_{2}\right)$, 50.93 (benzyl- $\mathrm{NCH}_{2} \mathrm{CH}_{2} \mathrm{NCH}_{2} \mathrm{CH}_{2}$ ), 51.68 (benzyl- $\mathrm{NCH}_{2}$ $\mathrm{CH}_{2} \mathrm{NCH}_{2}$ ), 55.12 (benzyl- $\left.\mathrm{NCH}_{2} \mathrm{CH}_{2}\right)$, $59.52\left(\mathrm{PhCH}_{2}-\right)$, $127.42\left(2 \times \mathrm{SO}_{2} \mathrm{CCHCH}\right), 127.57\left(\mathrm{CH}_{2} \mathrm{CCHCHCH}\right)$, $128.25\left(\mathrm{CH}_{2} \mathrm{CCHCH}\right), 129.70\left(2 \times \mathrm{SO}_{2} \mathrm{CCHCH}\right), 129.92$ $\left(\mathrm{CH}_{2} \mathrm{CCHCH}\right), 134.68\left(2 \times \mathrm{SO}_{2} \mathrm{CCH}\right), 135.62\left(\mathrm{SO}_{2} \mathrm{CCH}\right)$, $136.31\left(\mathrm{CH}_{2} \mathrm{CCH}\right), 143.45\left(\mathrm{SO}_{2} \mathrm{CCHCHC}\right), 143.58(2 \times$ $\mathrm{SO}_{2} \mathrm{CCHCHC}$ ). $-\mathrm{C}_{36} \mathrm{H}_{44} \mathrm{~N}_{4} \mathrm{O}_{6} \mathrm{~S}_{3}$ (724.95): calcd. C 59.65, H 6.12, N 7.73, S 13.27; found C 59.08, H 6.14, N 7.94, $\mathrm{S} 13.22$.

$\mathrm{C}_{6} \mathrm{H}_{5} \mathrm{CH}_{2} \mathrm{~N}\left(\mathrm{CH}_{2} \mathrm{CH}_{2} \mathrm{NH}\right)_{3} \mathrm{CH}_{2} \mathrm{CH}_{2}$ (6). Route I: $5.8 \mathrm{~g}$ ( $8.0 \mathrm{mmol})$ of $5,6.8 \mathrm{~g}(48 \mathrm{mmol})$ of anhydrous $\mathrm{Na}_{2} \mathrm{HPO}_{4}$, and sodium amalgam $(2 \%, 9.5 \mathrm{~g}, 48 \mathrm{mmol})$ were stirred in $\mathrm{CH}_{3} \mathrm{CN}(250 \mathrm{~mL})$ at $80{ }^{\circ} \mathrm{C}$ for one day. The colour- less mixture changed to white, and mercury precipitated which was separated. The solvent was removed on a rotary evaporator and the grey residue was dissolved in $\mathrm{CHCl}_{3}$ $(80 \mathrm{~mL})$ and washed three times with water $(55 \mathrm{~mL})$. The organic phases were combined and dried with $\mathrm{Na}_{2} \mathrm{SO}_{4}$. The solvent was removed and the crude product was dried under vacuum. Recrystallization from $\mathrm{CH}_{2} \mathrm{Cl}_{2} / \mathrm{CH}_{3} \mathrm{OH}$ $(10: 1)$ yielded 6 as a bright yellow solid. M.p. 83$85{ }^{\circ} \mathrm{C}$. Yield: $1.6 \mathrm{~g}(80 \%)$. Route II: $5.1 \mathrm{~g}(14 \mathrm{mmol})$

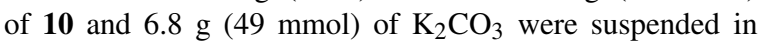
DMF $(150 \mathrm{~mL})$ and stirred for $30 \mathrm{~min}$ at $75{ }^{\circ} \mathrm{C}$. Afterwards $\mathrm{C}_{6} \mathrm{H}_{5} \mathrm{CH}_{2} \mathrm{Br}(1.7 \mathrm{~mL}, 14 \mathrm{mmol})$ was added dropwise and the mixture was refluxed for $2 \mathrm{~h}$ precipitating a white solid. After cooling to r.t., filtering and evaporating the solvent, the yellow residue was treated with $\mathrm{HCl}$ $(35 \mathrm{~mL}, 10 \%)$ and stirred at r.t. on air for further $16 \mathrm{~h}$. After raising the $\mathrm{pH}$ to 8 a brown solid was formed and removed by centrifugation. The resulting clear blue solution was extracted with $\mathrm{CHCl}_{3}(4 \times 35 \mathrm{~mL})$. The combined organic layers were dried with $\mathrm{Na}_{2} \mathrm{SO}_{4}$. A yellow solid was obtained after evaporation of all volatiles and drying under vacuum. Yield: $2.49 \mathrm{~g}(68 \%) .-{ }^{1} \mathrm{H}$ NMR $\left(25{ }^{\circ} \mathrm{C}, 400 \mathrm{MHz}, \mathrm{CDCl}_{3}\right): \delta=2.44-2.77(\mathrm{~m}, 16 \mathrm{H}$, macrocyclic $\left.\mathrm{CH}_{2}\right), 3.52\left(\mathrm{~s}, 2 \mathrm{H}, \mathrm{PhCH}_{2}\right), 7.12-7.25(\mathrm{~m}$, $5 \mathrm{H}$, benzyl-H). $-{ }^{13} \mathrm{C}-\mathrm{NMR}\left(25^{\circ} \mathrm{C}, 100.64 \mathrm{MHz}, \mathrm{CDCl}_{3}\right)$ : $\delta=44.54$ (benzyl-N $\left(\mathrm{CH}_{2}\right)_{2} \mathrm{NCH}_{2} \mathrm{CH}_{2}$ ), 45.92 (benzyl$\mathrm{N}\left(\mathrm{CH}_{2}\right)_{2} \mathrm{NCH}_{2}$ ), 46.70 (benzyl- $\mathrm{NCH}_{2} \mathrm{CH}_{2}$ ), 50.90 (benzyl- $\left.\mathrm{NCH}_{2}\right), 58.91\left(\mathrm{PhCH}_{2}-\right), 126.82(\mathrm{CCHCHCH}), 128.06$ $(\mathrm{CCHCHCH}), 129.06(\mathrm{CCHCHCH}), 138.78(\mathrm{CCHCHCH}) .-$ $\mathrm{C}_{15} \mathrm{H}_{26} \mathrm{~N}_{4}$ (262.40): calcd. C 68.66, H 9.99, N 21.35; found C 68.28, H 9.80, N 21.28.

$p-\mathrm{CH}_{3} \mathrm{C}_{6} \mathrm{H}_{4} \mathrm{SO}_{2} \mathrm{~N}\left(\mathrm{CH}_{2} \mathrm{CH}_{2} \mathrm{NHSO}_{2} \mathrm{C}_{6} \mathrm{H}_{4} \mathrm{CH}_{3}-p\right)_{2}$ $2.0 \mathrm{~g}(19 \mathrm{mmol})$ of 1 and $\mathrm{NEt}_{3}(7.9 \mathrm{~mL}, 57 \mathrm{mmol})$ were dissolved in $\mathrm{CH}_{2} \mathrm{Cl}_{2}(100 \mathrm{~mL})$ and the mixture cooled to $-2{ }^{\circ} \mathrm{C}$. A solution of $p$-toluenesulfonylchloride $(10.9 \mathrm{~g}$, $57 \mathrm{mmol})$ in $\mathrm{CH}_{2} \mathrm{Cl}_{2}(50 \mathrm{~mL})$ was added dropwise keeping the temperature at $0{ }^{\circ} \mathrm{C}$. The mixture was stirred at this temperature for $24 \mathrm{~h}$ and then washed with water $(3 \times 55 \mathrm{~mL})$. The organic layer was dried with $\mathrm{Na}_{2} \mathrm{SO}_{4}$. Evaporation and drying under vacuum resulted in 7 as a white solid. M.p. $59-61{ }^{\circ} \mathrm{C}$. Yield: $10.2 \mathrm{~g}$ (95\%). ${ }^{1} \mathrm{H}$ NMR $\left(25{ }^{\circ} \mathrm{C}, 200 \mathrm{MHz},\left[\mathrm{D}_{6}\right] \mathrm{DMSO}\right): \delta=2.37(\mathrm{~s}$, $\left.9 \mathrm{H}, \mathrm{Ts}-\mathrm{CH}_{3}\right), 2.81-3.02\left(\mathrm{~m}, 8 \mathrm{H}, \mathrm{NHCH}_{2} \mathrm{CH}_{2} \mathrm{~N}\right), 5.37$ (br, $2 \mathrm{H}, \mathrm{NH}), 7.33-7.40\left(\mathrm{~m}, 6 \mathrm{H}, \mathrm{SO}_{2} \mathrm{CCHCH}\right), 7.54$ $\left(\mathrm{d},{ }^{3} J=8.2 \mathrm{~Hz}, 2 \mathrm{H}, \mathrm{SO}_{2} \mathrm{CCH}\right), 7.66\left(\mathrm{~m}, 6 \mathrm{H},\left(\mathrm{d},{ }^{3} J=\right.\right.$ $\left.8.2 \mathrm{~Hz}, 4 \mathrm{H}, \mathrm{NHSO}_{2} \mathrm{CCH}\right) .-{ }^{13} \mathrm{C} \mathrm{NMR}\left(25^{\circ} \mathrm{C}, 50.32 \mathrm{MHz}\right.$, [D $\mathrm{D}_{6}$ DMSO): $\delta=20.97\left(\mathrm{Ts}-\mathrm{CH}_{3}\right), 41.59\left(2 \times \mathrm{NHCH}_{2}\right)$, $48.40\left(2 \times \mathrm{NHCH}_{2} \mathrm{CH}_{2}\right), 126.54\left(2 \times \mathrm{NHSO}_{2} \mathrm{CCHCH}\right)$, $126.83\left(\mathrm{NSO}_{2} \mathrm{CCHCH}\right), 129.68\left(2 \times \mathrm{NHSO}_{2} \mathrm{CCHCH}\right)$, $129.88\left(\mathrm{NSO}_{2} \mathrm{CCHCH}\right), 135.31\left(\mathrm{NSO}_{2} \mathrm{CCH}\right), 137.36(2 \times$ $\left.\mathrm{NHSO}_{2} \mathrm{CCH}\right), 142.76\left(2 \times \mathrm{NHSO}_{2} \mathrm{CCHCHCCH}_{3}\right), 143.46$ $\left(\mathrm{NSO}_{2} \mathrm{CCHCHCCH}_{3}\right)$. $-\mathrm{C}_{25} \mathrm{H}_{31} \mathrm{~N}_{3} \mathrm{O}_{6} \mathrm{~S}_{3}$ (565.73): calcd. C 53.08, H 5.52, N 7.43, S 17.00; found C 53.15, H 5.45, 


\section{$\mathrm{N} 17.29, \mathrm{~S} 17.29$}

$\left(p-\mathrm{CH}_{3} \mathrm{C}_{6} \mathrm{H}_{4} \mathrm{SO}_{2} \mathrm{NCH}_{2} \mathrm{CH}_{2}\right)_{4}(8) . \mathrm{Cs}_{2} \mathrm{CO}_{3}(50.6 \mathrm{~g}, 0.150$ mol) was suspended in a solution of $7(29.3 \mathrm{~g}, 0.052 \mathrm{~mol})$ in $\mathrm{CH}_{3} \mathrm{CN}(300 \mathrm{~mL})$ and heated to $80{ }^{\circ} \mathrm{C}$. Afterwards $21.5 \mathrm{~g}(0.052 \mathrm{~mol})$ of 4 , dissolved in $\mathrm{CH}_{3} \mathrm{CN}(250 \mathrm{~mL})$, were added over a period of $1 \mathrm{~h}$ and the mixture stirred at this temperature for $2 \mathrm{~d}$. After cooling to r.t. $500 \mathrm{~mL}$ of water were added in order to separate the partially precipitated product from the excess carbonate by filtration. The remaining solution was extracted with $\mathrm{CH}_{2} \mathrm{Cl}_{2}(5 \times$ $80 \mathrm{~mL}$ ), the organic layers were combined, the solvent was reduced in volume to about one third and $\mathrm{CH}_{3} \mathrm{OH}(100 \mathrm{~mL})$ was added. After storing the solution for $3 \mathrm{~d}$ at $5{ }^{\circ} \mathrm{C}, \mathbf{8}$ was obtained as a white solid. Decomposition $>260{ }^{\circ} \mathrm{C}$. Yield: $27.9 \mathrm{~g}(68 \%) .-{ }^{1} \mathrm{H} \mathrm{NMR}\left(25^{\circ} \mathrm{C}, 200 \mathrm{MHz}, \mathrm{CDCl}_{3}\right)$ : $\delta=2.45\left(\mathrm{~s}, 12 \mathrm{H}, \mathrm{Ts}-\mathrm{CH}_{3}\right), 3.43\left(\mathrm{~s}, 16 \mathrm{H}\right.$, macrocyclic $\left.\mathrm{CH}_{2}\right)$, $7.28-7.35\left(\mathrm{~m}, 8 \mathrm{H}, \mathrm{SO}_{2} \mathrm{CCHCH}\right), 7.61-7.71(\mathrm{~m}, 8 \mathrm{H}$, $\left.\mathrm{SO}_{2} \mathrm{CCHCH}\right) .-{ }^{13} \mathrm{C} \mathrm{NMR}\left(25^{\circ} \mathrm{C}, 50.32 \mathrm{MHz}, \mathrm{CDCl}_{3}\right): \delta=$ $21.38\left(\mathrm{Ts}-\mathrm{CH}_{3}\right), 44.41\left(\mathrm{CH}_{2}\right), 126.43\left(\mathrm{SO}_{2} \mathrm{CCHCH}\right), 129.68$ $\left(\mathrm{SO}_{2} \mathrm{CCHCH}\right), 135.41\left(\mathrm{SO}_{2} \mathrm{CCH}\right), 142.75\left(\mathrm{SO}_{2} \mathrm{CCHCHC}\right)$. $-\mathrm{C}_{36} \mathrm{H}_{44} \mathrm{~N}_{4} \mathrm{O}_{8} \mathrm{~S}_{4}$ (789.01): calcd. C 54.80, H 5.62, N 7.10, S 16.25; found C 54.45, H 5.44, N 7.29, S 16.00.

$\left(\mathrm{HNCH}_{2} \mathrm{CH}_{2}\right)_{4}(\mathbf{9}) .65 .3 \mathrm{~g}(0.083 \mathrm{~mol})$ of 8 was stirred with $100 \mathrm{~mL}$ of concentrated sulphuric acid for $3 \mathrm{~d}$ at $130{ }^{\circ} \mathrm{C}$. The initially colorless solution changed to brown after a few $\mathrm{h}$ and a black precipitate occurred. The mixture was cooled to $0{ }^{\circ} \mathrm{C}$, diluted with $150 \mathrm{~mL}$ of water and then the $\mathrm{pH}$ was adjusted to $>13$ by addition of solid $\mathrm{KOH} \mathrm{(130} \mathrm{g,}$ $2.32 \mathrm{~mol})$. The filtered precipitate was washed with $\mathrm{EtOH}$ $(2 \times 90 \mathrm{~mL})$ and the aqueous and the organic phases were combined and evaporated. The brownish residue was dissolved in $80 \mathrm{~mL}$ of $0.1 \mathrm{M} \mathrm{HCl}$ and washed with $\mathrm{CH}_{2} \mathrm{Cl}_{2}(4 \times$ $30 \mathrm{~mL}$ ). The $\mathrm{pH}$ of the aqueous phase was adjusted again to $>13$ and the solution extracted with $\mathrm{CHCl}_{3}(4 \times 30 \mathrm{~mL})$. Af ter combining and drying of the organic phases with $\mathrm{K}_{2} \mathrm{CO}_{3}$ the solvent was removed and the white solid of $\mathbf{9}$ was dried in a vacuum. M.p. $113-114{ }^{\circ} \mathrm{C}$. Yield: $8.6 \mathrm{~g}(60 \%) .-{ }^{1} \mathrm{H}$ NMR $\left(25{ }^{\circ} \mathrm{C}, 400 \mathrm{MHz}, \mathrm{CDCl}_{3}\right): \delta=2.17(\mathrm{~s}, 4 \mathrm{H}, \mathrm{NH}), 2.68(\mathrm{~s}$, $\left.16 \mathrm{H}, \mathrm{CH}_{2}\right) .-{ }^{13} \mathrm{C} \mathrm{NMR}\left(25{ }^{\circ} \mathrm{C}, 100.64 \mathrm{MHz}, \mathrm{CDCl}_{3}\right): \delta=$ $46.12\left(\mathrm{CH}_{2}\right) .-\mathrm{C}_{8} \mathrm{H}_{20} \mathrm{~N}_{4}$ (127.27): calcd. C 55.78, H 11.70, N 32.52; found C 55.13, H 11.99, N 32.50.

$(\mathrm{CO})_{3} \mathrm{Mo}\left(\mathrm{HNCH}_{2} \mathrm{CH}_{2}\right)_{4}$ (10). 2.7. $\mathrm{g}(16 \mathrm{mmol})$ of 9 and $4.6 \mathrm{~g}(16 \mathrm{mmol})$ of $\mathrm{Mo}(\mathrm{CO})_{6}$ were suspended in $n$-dibutylether $(80 \mathrm{~mL})$ and heated to $140{ }^{\circ} \mathrm{C}$ for $2 \mathrm{~h}$. The yellow precipitate was filtered off and washed with diethyl ether $(3 \times 15 \mathrm{~mL})$ to yield $5.2 \mathrm{~g}(92 \%)$ of $\mathbf{1 0} .-\mathrm{C}_{11} \mathrm{H}_{20} \mathrm{~N}_{4} \mathrm{O}_{3} \mathrm{Mo}$ (352.24): calcd. C 37.51, H 5.72, N 15.91; found C 36.93, $\mathrm{H}$ 5.83, N 15.61.

$\mathrm{C}_{2} \mathrm{H}_{5} \mathrm{OC}(\mathrm{O}) \mathrm{CH}_{2} \mathrm{~N}\left(\mathrm{CH}_{2} \mathrm{CH}_{2} \mathrm{NH}\right)_{3} \mathrm{CH}_{2} \mathrm{CH}_{2}$ (11). 11 was prepared in analogy to the synthesis of $\mathbf{6}$ following route II using $1.1 \mathrm{~g}(3.0 \mathrm{mmol})$ of $\mathbf{1 0}, 1.4 \mathrm{~g}(49 \mathrm{mmol})$ of $\mathrm{K}_{2} \mathrm{CO}_{3}$, $0.33 \mathrm{~mL}$ ( $3.0 \mathrm{mmol})$ of $\mathrm{BrCH}_{2} \mathrm{COOEt}$, and $80 \mathrm{~mL}$ of DMF. Yield: $0.40 \mathrm{~g}(55 \%)$ of $\mathbf{1 1}$ as a light yellow solid. M.p.
89-91 ${ }^{\circ} \mathrm{C} .-{ }^{1} \mathrm{H}$ NMR $\left(25{ }^{\circ} \mathrm{C}, 400 \mathrm{MHz}, \mathrm{CDCl}_{3}\right): \delta=$ $1.20\left(\mathrm{t},{ }^{3} \mathrm{~J}=8.9 \mathrm{~Hz}, 3 \mathrm{H}, \mathrm{CH}_{2} \mathrm{CH}_{3}\right), 2.48-2.84(\mathrm{~m}, 16 \mathrm{H}$, macrocyclic $\mathrm{CH}_{2}$ ), 3.30 (s, $\left.2 \mathrm{H}, \mathrm{NCH}_{2} \mathrm{CO}\right), 4.10$ (q, ${ }^{3} \mathrm{~J}=$ $\left.8.9 \mathrm{~Hz}, 2 \mathrm{H}, \mathrm{CH}_{2} \mathrm{CH}_{3}\right) .-{ }^{13} \mathrm{C} \mathrm{NMR}\left(25{ }^{\circ} \mathrm{C}, 100.64 \mathrm{MHz}\right.$, $\left.\mathrm{CDCl}_{3}\right): \delta=13.95\left(\mathrm{CH}_{2} \mathrm{CH}_{3}\right), 51.05\left(\mathrm{NCH}_{2} \mathrm{CO}\right), 46.30$ (ester- $\left.\mathrm{N}\left(\mathrm{CH}_{2}\right)_{2} \mathrm{NCH}_{2} \mathrm{CH}_{2}\right), 47.03$ (ester- $\mathrm{N}\left(\mathrm{CH}_{2}\right)_{2} \mathrm{NCH}_{2}$ ), 50.34 (ester- $\left.\mathrm{NCH}_{2} \mathrm{CH}_{2}\right), \quad 55.70$ (ester- $\left.\mathrm{NCH}_{2}\right), \quad 60.65$ $\left(\mathrm{CH}_{2} \mathrm{CH}_{3}\right), 172.34\left(\mathrm{NCH}_{2} \mathrm{CO}\right) .-\mathrm{C}_{12} \mathrm{H}_{26} \mathrm{~N}_{4} \mathrm{O}_{2}(258,36)$ : calcd. C 55.79, H 10.14, N 21.69; found C 55.95, H 10.30, N 21.80.

$\mathrm{C}_{6} \mathrm{H}_{5} \mathrm{CH}_{2} \mathrm{NCH}_{2} \mathrm{CH}_{2}\left[\mathrm{~N}\left(\mathrm{CH}_{2} \mathrm{COO}^{t} \mathrm{Bu}\right) \mathrm{CH}_{2} \mathrm{CH}_{2}\right]_{3}$ (12). $1.1 \mathrm{~g}(4 \mathrm{mmol})$ of 6 and $1.7 \mathrm{~g}(12 \mathrm{mmol})$ of dried $\mathrm{K}_{2} \mathrm{CO}_{3}$ were suspended in DMF $(180 \mathrm{~mL})$ and heated to $80{ }^{\circ} \mathrm{C}$ for $30 \mathrm{~min}$. Afterwards $\mathrm{BrCH}_{2} \mathrm{COO}^{t} \mathrm{Bu}(2.34 \mathrm{~mL}, 12 \mathrm{mmol})$ was added dropwise and the mixture refluxed for $20 \mathrm{~h}$, precipitating $\mathrm{KBr}$ as a white solid. The solvent was evaporated, the residue dissolved in $\mathrm{CH}_{2} \mathrm{Cl}_{2}(50 \mathrm{~mL})$ and filtered. The colorless solution was then washed with water $(3 \times 45 \mathrm{~mL})$ and the organic layer was dried with $\mathrm{Na}_{2} \mathrm{SO}_{4}$. A yellow solid was obtained after evaporation of the volatiles and drying in vacuum. Yield: $(1.81 \mathrm{~g}, 75 \%)$. M.p. $95-98{ }^{\circ} \mathrm{C} .-{ }^{1} \mathrm{H}$ NMR $\left(25^{\circ} \mathrm{C}, 200 \mathrm{MHz}, \mathrm{CDCl}_{3}\right): \delta=1.39\left(\mathrm{~s}, 18 \mathrm{H},{ }^{t} \mathrm{Bu}\right), 1.43(\mathrm{~s}$, $\left.9 \mathrm{H},{ }^{t} \mathrm{Bu}\right), 2.57-2.81\left(\mathrm{~m}, 16 \mathrm{H}\right.$, macrocyclic $\left.\mathrm{CH}_{2}\right), 3.17$ (s, $4 \mathrm{H}, \mathrm{CH}_{2} \mathrm{CO}_{2}^{t} \mathrm{Bu}$ ), 3.29 (s, $\left.2 \mathrm{H}, \mathrm{CH}_{2} \mathrm{CO}_{2}^{t} \mathrm{Bu}\right), 3.49$ (s, $2 \mathrm{H}$, benzyl- $\left.\mathrm{CH}_{2}\right), 7.20-7.25(\mathrm{~m}, 5 \mathrm{H}$, benzyl- $\mathrm{H}) .-{ }^{13} \mathrm{C}$ NMR $\left.\left(25{ }^{\circ} \mathrm{C}, 50.32 \mathrm{MHz}, \mathrm{CDCl}_{3}\right): \delta=28.72\left(\mathrm{C} \mathrm{CH}_{3}\right)_{3}\right), 52.41$ (benzyl- $\mathrm{N}\left(\mathrm{CH}_{2}\right)_{2} \mathrm{NCH}_{2} \mathrm{CH}_{2}$ ), 52.60 (benzyl- $\mathrm{NCH}_{2} \mathrm{CH}_{2}$ ), 52.72 (benzyl- $\left.\mathrm{NCH}_{2} \mathrm{CH}_{2}\right), 56.68\left(\mathrm{NCH}_{2} \mathrm{CO}\right), 60.11\left(\mathrm{NCH}_{2}-\right.$

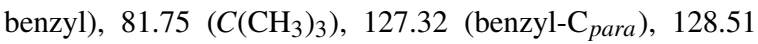

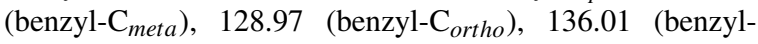
$\mathrm{C}_{\text {quart }}$ ), $169.68\left(\mathrm{NCH}_{2} \mathrm{CO}_{2}{ }^{t} \mathrm{Bu}\right) .-\mathrm{C}_{33} \mathrm{H}_{56} \mathrm{~N}_{4} \mathrm{O}_{6}$ (604.82): calcd. C 65.53, H 9.33, N 9.26; found C 65.83, H 9.51, N 9.40 .

$\mathrm{HNCH}_{2} \mathrm{CH}_{2}\left[\mathrm{~N}\left(\mathrm{CH}_{2} \mathrm{COO}^{t} \mathrm{Bu}\right) \mathrm{CH}_{2} \mathrm{CH}_{2}\right]_{3}$ (13). Hydrogen gas was bubbled through a suspension of 12 (1.2 g, $2.0 \mathrm{mmol})$ and the catalyst $\mathrm{Pd} / \mathrm{C}(10 \% \mathrm{Pd}, 200 \mathrm{mg})$ in a mixture of $\mathrm{CH}_{3} \mathrm{OH}$ and THF $(1: 1,300 \mathrm{~mL})$ at r. t. over night. After removing the catalyst by filtration over celite, the solvent was evaporated and the brownish residue dried in vacuum. Crystallization from a mixture of acetone/diisopropylether (2:1) yielded a light yellow solid (0.41 g, 40\%). M.p. $47-50{ }^{\circ} \mathrm{C}$. - IR (KBr): $v=1733$ (s, C=O, ester), 1158 (s, $\mathrm{C}-\mathrm{O}$, ester), $1059(\mathrm{~m}, \mathrm{C}-\mathrm{N}) \mathrm{cm}^{-1} .-{ }^{1} \mathrm{H}$ NMR $(400 \mathrm{MHz}$, $\mathrm{CDCl}_{3}$, r. t.): $\delta=1.39$ (s, $\left.27 \mathrm{H},{ }^{t} \mathrm{Bu}\right), 2.52(\mathrm{~m}, 4 \mathrm{H}$, $\left.\mathrm{NHCH}_{2} \mathrm{CH}_{2}\right), 2.68\left(\mathrm{~s}, 8 \mathrm{H}, \mathrm{NRCH}_{2} \mathrm{CH}_{2} \mathrm{NR}\right), 2.77$ (m, $4 \mathrm{H}$, $\left.\mathrm{NHCH}_{2}\right), 3.26\left(\mathrm{~s}, 6 \mathrm{H}, \mathrm{CH}_{2} \mathrm{CO}_{2}{ }^{t} \mathrm{Bu}\right) .-{ }^{13} \mathrm{C}\left\{{ }^{1} \mathrm{H}\right\} \mathrm{NMR}$ $\left(100.64 \mathrm{MHz}, \mathrm{CDCl}_{3}\right.$, r.t. $): \delta=28.24\left(\mathrm{C}\left(\mathrm{CH}_{3}\right)_{3}\right), 47.56$ $\left(\mathrm{NHCH}_{2} \mathrm{CH}_{2}\right), 50.70\left(\mathrm{NH}\left(\mathrm{CH}_{2}\right)_{2} \mathrm{NRCH}_{2}\right), 52.16\left(\mathrm{NHCH}_{2}\right)$, $52.24 \quad\left(\mathrm{NH}\left(\mathrm{CH}_{2}\right)_{2} \mathrm{NRCH}_{2} \mathrm{CH}_{2}\right), \quad 52.80 \quad\left(\mathrm{NH}\left(\mathrm{CH}_{2}\right)_{2} \mathrm{~N}-\right.$ $\left.\mathrm{R}\left(\mathrm{CH}_{2}\right)_{2} \mathrm{NCH}_{2} \mathrm{CO}\right), 57.23$ (s, $\left.\mathrm{NH}\left(\mathrm{CH}_{2}\right)_{2} \mathrm{NCH}_{2} \mathrm{CO}\right), 81.78$ $\left(\mathrm{C}\left(\mathrm{CH}_{3}\right)_{3}\right), 171.14\left(\mathrm{NCH}_{2} \mathrm{CO}\right)$. - MS (EI, $\left.70 \mathrm{eV}\right): \mathrm{m} / \mathrm{z}$ $(\%)=514(24.10)[\mathrm{M}]^{+}, 413(100)\left[\mathrm{M}-\mathrm{CO}_{2}-{ }^{t} \mathrm{Bu}\right]^{+}$. $\mathrm{C}_{26} \mathrm{H}_{50} \mathrm{~N}_{4} \mathrm{O}_{6}$ (514.70): calcd. C 60.67, H 9.79, N 10.89; 
found $\mathrm{C} 60.38, \mathrm{H} 9.62, \mathrm{~N} 10.39$.

$\mathrm{EtOC}(\mathrm{O}) \mathrm{CH}_{2} \mathrm{NCH}_{2} \mathrm{CH}_{2}\left[\mathrm{~N}\left(\mathrm{CH}_{2} \mathrm{COO}^{t} \mathrm{Bu}\right) \mathrm{CH}_{2} \mathrm{CH}_{2}\right]_{3}$ (14) was prepared as described for 12 from $11(0.25 \mathrm{~g}$, $1.0 \mathrm{mmol}), \mathrm{K}_{2} \mathrm{CO}_{3}(0.41 \mathrm{~g}, 3.0 \mathrm{mmol}), \mathrm{BrCH}_{2} \mathrm{COO}^{t} \mathrm{Bu}$ $(0.44 \mathrm{~mL}, 3.0 \mathrm{mmol})$, and $25 \mathrm{~mL}$ of $\mathrm{DMF}$ as a white solid. Yield: $0.44 \mathrm{~g}(74 \%)$. Decomposition $>140{ }^{\circ} \mathrm{C}$. ${ }^{1} \mathrm{H} \mathrm{NMR}\left(200 \mathrm{MHz}, \mathrm{CDCl}_{3}\right.$, r. t.): $\delta=1.21\left(\mathrm{t},{ }^{3} J=8.85 \mathrm{~Hz}\right.$, $\left.3 \mathrm{H}, \mathrm{OCH}_{2} \mathrm{CH}_{3}\right), 1.39\left(\mathrm{~s}, 18 \mathrm{H},{ }^{t} \mathrm{Bu}\right), 1.41\left(\mathrm{~s}, 9 \mathrm{H},{ }^{t} \mathrm{Bu}\right)$, 2.50-2.86 (m, $16 \mathrm{H}$, macrocyclic $\left.\mathrm{CH}_{2}\right), 3.20-3.31(\mathrm{~m}$, $\left.6 \mathrm{H}, \mathrm{CH}_{2} \mathrm{CO}_{2}{ }^{t} \mathrm{Bu}\right), 3.34$ (s, $\left.2 \mathrm{H}, \mathrm{NCH}_{2} \mathrm{CO}_{2} \mathrm{Et}\right), 4.10$ (q, ${ }^{3} \mathrm{~J}=$ $\left.8.85 \mathrm{~Hz}, 2 \mathrm{H}, \mathrm{OCH}_{2} \mathrm{CH}_{3}\right) .-{ }^{13} \mathrm{C}\left\{{ }^{1} \mathrm{H}\right\} \mathrm{NMR}(50.32 \mathrm{MHz}$, $\mathrm{CDCl}_{3}$, r. t. $): \delta=14.03\left(\mathrm{OCH}_{2} \mathrm{CH}_{3}\right), 27.72\left(\mathrm{C}_{\left.\left(\mathrm{CH}_{3}\right)_{3}\right), 51.59}\right.$ $\left(\mathrm{NCH}_{2} \mathrm{CO}\right), 55.91\left(\mathrm{EtO}_{2} \mathrm{CCH}_{2} \mathrm{~N}\left(\mathrm{CH}_{2}\right)_{2} \mathrm{NCH}_{2} \mathrm{CH}_{2}\right), 56.25$ $\left(\mathrm{EtO}_{2} \mathrm{CCH}_{2} \mathrm{~N}\left(\mathrm{CH}_{2}\right)_{2} \mathrm{NCH}_{2}\right), 56.41\left(\mathrm{EtO}_{2} \mathrm{CCH}_{2} \mathrm{NCH}_{2} \mathrm{CH}_{2}\right)$, $56.78 \quad\left(\mathrm{EtO}_{2} \mathrm{CCH}_{2} \mathrm{NCH}_{2}\right), \quad 60.85 \quad\left(\mathrm{OCH}_{2} \mathrm{CH}_{3}\right), \quad 81.75$ $\left(C\left(\mathrm{CH}_{3}\right)_{3}\right), 171.98\left(\mathrm{NCH}_{2} \mathrm{CO}_{2}{ }^{t} \mathrm{Bu}\right), 172.64\left(\mathrm{NCH}_{2} \mathrm{CO}_{2} \mathrm{Et}\right)$. $-\mathrm{C}_{30} \mathrm{H}_{56} \mathrm{~N}_{4} \mathrm{O}_{8}$ (600.80): calcd. C 59.98, H 9.39, N 9.33; found $\mathrm{C}$ 59.83, H 9.31, N 9.20.

$\mathrm{HOC}(\mathrm{O}) \mathrm{CH}_{2} \mathrm{NCH}_{2} \mathrm{CH}_{2}\left[\mathrm{~N}\left(\mathrm{CH}_{2} \mathrm{COO}^{t} \mathrm{Bu}\right) \mathrm{CH}_{2} \mathrm{CH}_{2}\right]_{3}$ (15). a) 15 was prepared as described above for $\mathbf{1 2}$ from $\mathbf{1 3}$ (0.1 g, $0.2 \mathrm{mmol}$ ), $\mathrm{K}_{2} \mathrm{CO}_{3}$ (28 mg, $0.2 \mathrm{mmol}$ ), $\mathrm{BrCH}_{2} \mathrm{COOH}$ $(0.014 \mathrm{~mL}, 0.2 \mathrm{mmol})$ and $10 \mathrm{~mL}$ of DMF at $70{ }^{\circ} \mathrm{C}(2 \mathrm{~h})$, as a white solid. Yield: $74 \mathrm{mg}(65 \%)$. M.p. $127-130{ }^{\circ} \mathrm{C}$. b) $\mathbf{1 4}(0.3 \mathrm{~g}, 0.5 \mathrm{mmol})$ was suspended in aqueous $\mathrm{KOH}$ solution $(1 \mathrm{M}, 5 \mathrm{~mL})$ and stirred for one day at $30^{\circ} \mathrm{C}$. The mixture was brought to dryness, the residue suspended in $\mathrm{C}_{2} \mathrm{H}_{5} \mathrm{OH}(10 \mathrm{~mL})$ and then filtered. This process was repeated five times. The combined organic layers were dried with $\mathrm{Na}_{2} \mathrm{SO}_{4}$. A white microcrystalline solid was obtained after evaporation of the solvents and drying in vacuum. Yield: $0.17 \mathrm{~g}(59 \%)$. M.p. $129-131{ }^{\circ} \mathrm{C}$. - IR (KBr): $v=1738$ (s, C=O, ester), 1644 (s, C=O, acid), 1161 (s, C-O, ester), $1120(\mathrm{~m}, \mathrm{C}-\mathrm{N}) \mathrm{cm}^{-1} .-{ }^{1} \mathrm{H}$ NMR (400 MHz, $\mathrm{CDCl}_{3}$, r. t.): $\delta=1.42\left(\mathrm{~s}, 27 \mathrm{H},{ }^{t} \mathrm{Bu}\right), 2.76$ (s, $8 \mathrm{H}$, acid- $\left.\mathrm{N}\left(\mathrm{CH}_{2}\right)_{2} \mathrm{NCH}_{2} \mathrm{CH}_{2}\right), 3.04\left(\mathrm{~m}, 4 \mathrm{H}\right.$, acid- $\left.\mathrm{NCH}_{2} \mathrm{CH}_{2}\right)$, 3.29 (s, $4 \mathrm{H}$, acid- $\left.\mathrm{N}\left(\mathrm{CH}_{2}\right)_{2} \mathrm{NCH}_{2} \mathrm{CO}\right), 3.37$ (s, $2 \mathrm{H}$, acid$\left.\mathrm{N}\left\{\left(\mathrm{CH}_{2}\right)_{2} \mathrm{~N}\right\}_{2} \mathrm{CH}_{2} \mathrm{CO}\right), 3.60\left(\mathrm{~m}, 4 \mathrm{H}\right.$, acid- $\left.\mathrm{NCH}_{2}\right), 3.69$ (s, $\left.2 \mathrm{H}, \mathrm{CH}_{2} \mathrm{COOH}\right) .-{ }^{13} \mathrm{C}\left\{{ }^{1} \mathrm{H}\right\} \mathrm{NMR}\left(100.64 \mathrm{MHz}, \mathrm{CDCl}_{3}\right.$, r.t.): $\delta=28.13\left(\mathrm{C}\left(\mathrm{CH}_{3}\right)_{3}\right), 48.47$ (acid- $\left.\mathrm{NCH}_{2} \mathrm{CH}_{2}\right), 50.28$ (acid- $\mathrm{N}\left(\mathrm{CH}_{2}\right)_{2} \mathrm{NCH}_{2}$ ), 53.51 (acid- $\mathrm{NCH}_{2} \mathrm{CH}_{2} \mathrm{NCH}_{2} \mathrm{CH}_{2}$ ), $55.75\left(\mathrm{NCH}_{2} \mathrm{COOH}\right), 56.07$ (acid- $\mathrm{N}\left\{\left(\mathrm{CH}_{2}\right)_{2} \mathrm{~N}\right\}_{2} \mathrm{CH}_{2} \mathrm{CO}$ ), 56.75 (acid-N $\left.\left(\mathrm{CH}_{2}\right)_{2} \mathrm{NCH}_{2} \mathrm{CO}\right), 81.80\left(\mathrm{C}\left(\mathrm{CH}_{3}\right)_{3}\right), 166.95$ $(\mathrm{COOH}), 169.93$ (acid- $\left.\mathrm{N}\left(\mathrm{CH}_{2}\right)_{2} \mathrm{NCH}_{2} \mathrm{CO}\right), 170.69$ (acid$\left.\mathrm{N}\left\{\left(\mathrm{CH}_{2}\right)_{2} \mathrm{~N}\right\}_{2} \mathrm{CH}_{2} \mathrm{CO}\right) .-\mathrm{MS}(\mathrm{EI}, 70 \mathrm{eV}): \mathrm{m} / z(\%)=572$ (3.10) $[\mathrm{M}]^{+}, 471(100)\left[\mathrm{M}-\mathrm{CO}_{2}-{ }^{t} \mathrm{Bu}\right]^{+} .-\mathrm{C}_{28} \mathrm{H}_{52} \mathrm{~N}_{4} \mathrm{O}_{8}$ (572.74): calcd. C 58.72, H 9.15, N 9.78; found C 58.61, $\mathrm{H} 9.07$, N 9.51.

$\mathrm{H}_{2} \mathrm{~N}\left(\mathrm{CH}_{2}\right)_{4} \mathrm{NHC}(\mathrm{O}) \mathrm{CH}_{2} \mathrm{NCH}_{2} \mathrm{CH}_{2}\left[\mathrm{~N}\left(\mathrm{CH}_{2} \mathrm{COOH}\right) \mathrm{CH}_{2}^{-}\right.$ $\mathrm{CH}_{2} \mathrm{~J}_{3}(\mathbf{1 6})$. To a solution of $\mathbf{1 5}(1.34 \mathrm{~g}, 2.35 \mathrm{mmol})$ in DMF $(40 \mathrm{~mL}), 0.983 \mathrm{~g}(2.585 \mathrm{mmol})$ of HATU and $0.5 \mathrm{~mL}$ of Hünig's base were added. The mixture was stirred for $5 \mathrm{~min}$ and added to $5 \mathrm{~g}$ of 1,4-diaminobutane trityl resin (loading $0.47 \mathrm{mmol} / \mathrm{g}, 2.35 \mathrm{mmol}$ ) in DMF. The reaction mixture was agitated at r.t. overnight and the solvent removed in vacuum. Afterwards the cleavage from the resin was carried out with $50 \mathrm{~mL}$ of TFA, $5 \%$ water and $1 \%$ tri-iso-propylsilane for $2 \mathrm{~h}$. The mixture was filtered and the filtrate was evaporated in vacuum. The residue was washed with ether yielding 16 (935 mg, 84\%). Further purification was achieved by preparative HPLC (Agilent-Prep-C18 column; solvent A: $0.1 \%$ TFA in water; solvent $\mathrm{B}: 10 \%$ of aq. $0.1 \%$ TFA, $90 \%$ aq. $\mathrm{CH}_{3} \mathrm{CN}$ ). Removal of the mobile phase gave the product as a lyophilized solid. M.p. $168-170{ }^{\circ} \mathrm{C}$. ${ }^{1} \mathrm{H} \mathrm{NMR}\left(400 \mathrm{MHz}, \mathrm{D}_{2} \mathrm{O}\right): \delta=1.40\left(\mathrm{~m}, 2 \mathrm{H}, \mathrm{NH}_{2} \mathrm{CH}_{2} \mathrm{CH}_{2}\right)$, $1.48\left(\mathrm{~m}, 2 \mathrm{H}, \mathrm{NHCH}_{2} \mathrm{CH}_{2}\right), 2.89\left(\mathrm{t}, 2 \mathrm{H}, \mathrm{NH}_{2} \mathrm{CH}_{2}\right), 3.08(\mathrm{~m}$, $2 \mathrm{H}, \mathrm{CONHCH}_{2}$ ), 2.70-3.50 (broad, $16 \mathrm{H}, \mathrm{NCH}_{2} \mathrm{CH}_{2} \mathrm{~N}$ ), 3.60-4.20 (broad, $8 \mathrm{H}, \mathrm{NHCH}_{2} \mathrm{CO}$ ). $-{ }^{13} \mathrm{C}\left\{{ }^{1} \mathrm{H}\right\} \mathrm{NMR}$ $\left(100.64 \mathrm{MHz}, \quad \mathrm{D}_{2} \mathrm{O}\right): \quad \delta=24.2\left(\mathrm{NHCH}_{2} \mathrm{CH}_{2}\right), 25.4$ $\left(\mathrm{NH}_{2} \mathrm{CH}_{2} \mathrm{CH}_{2}\right), 38.6\left(\mathrm{CONHCH}_{2}\right), 39.1\left(\mathrm{NH}_{2} \mathrm{CH}_{2}\right), 47.0-$ 53.5 (broad, $\mathrm{NCH}_{2} \mathrm{CH}_{2} \mathrm{~N}$ ), $53.5-57.0$ (broad, $\mathrm{NHCH}_{2} \mathrm{CO}$ ), 174.0 - 175.0 (broad, CO) - MALDI-TOF MS: $\mathrm{m} / z=457$ $[\mathrm{M}+\mathrm{H}]^{+} .-\mathrm{C}_{20} \mathrm{H}_{38} \mathrm{~N}_{6} \mathrm{O}_{7}$ (474.56): calcd. C 50.62, H 8.07, $\mathrm{N} 17.71$; found $\mathrm{C} 50.53, \mathrm{H} 8.01, \mathrm{~N} 17.81$.

$\mathrm{C}_{4} \mathrm{H}_{2} \mathrm{O}_{2} \mathrm{~N}\left(\mathrm{CH}_{2}\right)_{2} \mathrm{C}(\mathrm{O}) \mathrm{NH}\left(\mathrm{CH}_{2}\right)_{4} \mathrm{NHC}(\mathrm{O}) \mathrm{CH}_{2} \mathrm{NCH}_{2}-$ $\mathrm{CH}_{2}\left[\mathrm{~N}\left(\mathrm{CH}_{2} \mathrm{COOH}\right) \mathrm{CH}_{2} \mathrm{CH}_{2}\right]_{3}$ (17). To a solution of $\mathbf{1 6}$ (500 mg, $1.05 \mathrm{mmol}$ ) in $25 \mathrm{~mL}$ of DMF, $0.75 \mathrm{~mL}^{\circ} \mathrm{NEt}_{3}$ and a solution of $560 \mathrm{mg}$ ( $2.1 \mathrm{mmol})$ of $\beta$-maleimidopropionic acid $\mathrm{N}$-hydroxysuccinimide ester in $10 \mathrm{~mL}$ of DMF were added. The mixture was allowed to stand for $4 \mathrm{~h}$ at r.t. with occasional stirring. The precipitate was filtered and the filtrate was evaporated to dryness. Impurities were removed by washing with $\mathrm{CHCl}_{3}$ and $\mathrm{CH}_{3} \mathrm{OH}$. Yield: $480 \mathrm{mg}$ (73\%) of 17. Further purification was achieved by preparative HPLC (Agilent-Prep-C18 column; solvent A: 0.1\% TFA in water; solvent B: $10 \%$ of aq. $0.1 \%$ TFA, $90 \%$ aq. $\left.\mathrm{CH}_{3} \mathrm{CN}\right)$. M.p. $183-185{ }^{\circ} \mathrm{C}$. $-{ }^{1} \mathrm{H}$ NMR (400 MHz, $\left.\mathrm{D}_{2} \mathrm{O}\right): \delta=1.42\left(\mathrm{~m}, 2 \mathrm{H}, \mathrm{NHCH}_{2} \mathrm{CH}_{2} \mathrm{CH}_{22}\right), 1.46(\mathrm{~m}$, $2 \mathrm{H}, \mathrm{NHCH}_{2} \mathrm{CH}_{2} \mathrm{CH}_{2}$ ), 2.38 (t, $2 \mathrm{H}, \mathrm{NCH}_{2} \mathrm{CH}_{2} \mathrm{CO}$ ), $3.05\left(\mathrm{t}, 2 \mathrm{H}, \mathrm{CONHCH}_{2}\right), 3.10\left(\mathrm{~m}, 2 \mathrm{H}, \mathrm{CONHCH}_{2}\right)$, $3.12-3.54$ (broad, $16 \mathrm{H}, \mathrm{NCH}_{2} \mathrm{CH}_{2} \mathrm{~N}$ ), 3.69 (t, $2 \mathrm{H}$, $\mathrm{NCH}_{2} \mathrm{CH}_{2} \mathrm{CO}$ ), $3.64-4.23$ (broad, $8 \mathrm{H}, \mathrm{NHCH}_{2} \mathrm{CO}$ ), 6.88 (s, $2 \mathrm{H}, \mathrm{CH}=\mathrm{CH}) .-{ }^{13} \mathrm{C}\left\{{ }^{1} \mathrm{H}\right\}$ NMR $\left(100.64 \mathrm{MHz}, \mathrm{D}_{2} \mathrm{O}\right)$ : $\delta=26.4\left(\mathrm{NHCH}_{2} \mathrm{CH}_{2} \mathrm{CH}_{2}\right), 27.1\left(\mathrm{NHCH}_{2} \mathrm{CH}_{2} \mathrm{CH}_{2}\right), 34.0$ $\left(\mathrm{NCH}_{2} \mathrm{CH}_{2} \mathrm{CO}\right), 37.9\left(\mathrm{NCH}_{2} \mathrm{CH}_{2} \mathrm{CO}\right), 39.6\left(\mathrm{CH}_{2} \mathrm{NHCO}\right)$, $40.2\left(\mathrm{CONHCH}_{2}\right), 47.0-54.0$ (broad, $\left.\mathrm{NCH}_{2} \mathrm{CH}_{2} \mathrm{~N}\right), 54.0-$ 57.0 (broad, $\left.\mathrm{NHCH}_{2} \mathrm{CO}\right), 135.6(\mathrm{CH}=\mathrm{CH}), 167.0-174.0$ (broad, CO) - MALDI-TOF MS: $m / z=626[\mathrm{M}+\mathrm{H}]^{+}$. $\mathrm{C}_{27} \mathrm{H}_{43} \mathrm{~N}_{7} \mathrm{O}_{10}$ (625.68): calcd. C 51.83, H 6.93, N 15.67; found $\mathrm{C}$ 51.23, $\mathrm{H}$ 7.12, $\mathrm{N} 15.71$.

$\left.\mathrm{LnC}_{4} \mathrm{H}_{2} \mathrm{O}_{2} \mathrm{~N}\left(\mathrm{CH}_{2}\right)_{2} \mathrm{ClO}\right) \mathrm{NH}\left(\mathrm{CH}_{2}\right)_{4} \mathrm{NHC}(\mathrm{O}) \mathrm{CH}_{2} \mathrm{NCH}_{2}-$ $\mathrm{CH}_{2}\left[\mathrm{~N}\left(\mathrm{CH}_{2} \mathrm{COO}\right) \mathrm{CH}_{2} \mathrm{CH}_{2}\right]_{3}$ (18)-(21). Using a $0.1 \mathrm{M}$ $\mathrm{Na}_{2} \mathrm{CO}_{3} / \mathrm{HCl}$ solution ( $\mathrm{pH}=7.5$ buffer), $0.15 \mathrm{mmol}$ of $\mathrm{YCl}_{3}, \mathrm{HoCl}_{3}, \mathrm{TmCl}_{3}$ or $\mathrm{LuCl}_{3}$ were dissolved and combined with $60 \mathrm{mg}(0.096 \mathrm{mmol})$ of 17. The $\mathrm{pH}$ was adjusted to 7.0 and the samples were kept at r.t. over night. Analytical HPLC was carried out to verify the 


\begin{tabular}{|c|c|c|}
\hline Compound & 3 & 7 \\
\hline Empirical formula & $\mathrm{C}_{25} \mathrm{H}_{31} \mathrm{~N}_{3} \mathrm{O}_{4} \mathrm{~S}_{2}$ & $\mathrm{C}_{25} \mathrm{H}_{31} \mathrm{~N}_{3} \mathrm{O}_{6} \mathrm{~S}_{3}$ \\
\hline Formula weight $\left[\mathrm{g} \mathrm{mol}^{-1}\right]$ & 501.65 & 565.71 \\
\hline Crystal size $\left[\mathrm{mm}^{3}\right]$ & $0.75 \times 0.52 \times 0.35$ & $0.52 \times 0.24 \times 0.15$ \\
\hline Crystal system & monoclinic & monoclinic \\
\hline Space group & $P 2_{1} / c$ & $P 2_{1} / n$ \\
\hline$Z$ & 4 & 4 \\
\hline$a[\AA]$ & $11.9855(4)$ & $5.1910(1)$ \\
\hline$b[\AA]$ & $10.9267(4)$ & $27.4576(5)$ \\
\hline$c[\AA]$ & $20.0058(7)$ & $18.9445(4)$ \\
\hline$\beta[\mathrm{deg}]$ & $97.530(1)$ & $93.168(1)$ \\
\hline$V\left[\AA^{3}\right]$ & $2597.40(16)$ & $2696.08(9)$ \\
\hline$D_{\text {calcd }}\left[\mathrm{g} \mathrm{cm}^{-3}\right]$ & 1.283 & 1.394 \\
\hline Absorption coefficient $\left[\mathrm{mm}^{-1}\right]$ & 0.240 & 0.320 \\
\hline Min./max. transmission & $0.9207 / 0.8404$ & $0.8880 / 0.4807$ \\
\hline$F(000)[\mathrm{e}]$ & 1064 & 1192 \\
\hline $2 \theta$ Range for data collection $[\mathrm{deg}]$ & $1.71 \leq \theta \leq 26.00$ & $1.48 \leq \theta \leq 27.50$ \\
\hline \multirow[t]{3}{*}{ Data set } & $-14 \leq h \leq 12$ & $-6 \leq h \leq 6$ \\
\hline & $-13 \leq k \leq 13$ & $-30 \leq k \leq 35$ \\
\hline & $-22 \leq l \leq 24$ & $-24 \leq l \leq 21$ \\
\hline Reflections, collected & $1756 \overline{4}-$ & $2046 \overline{7}$ \\
\hline Reflections, unique & $5095\left(R_{\text {int }}=0.0773\right)$ & $6177\left(R_{\text {int }}=0.0859\right)$ \\
\hline Data / restraints / parameter & $5095 / 0 / 317$ & $6177 / 1 / 345$ \\
\hline Goodness-of-Fit $\left(F^{2}\right)$ & 0.988 & 1.081 \\
\hline \multirow[t]{2}{*}{ Final $R$ indices $(I \geq 2 \sigma(I))$} & $R 1=0.0516$ & $R 1=0.0798$ \\
\hline & $w R 2=0.1180$ & $w R 2=0.1410$ \\
\hline \multirow[t]{2}{*}{$R$ indices (all data) } & $R 1=0.0971$ & $R 1=0.1404$ \\
\hline & $w R 2=0.1394$ & $w R 2=0.1625$ \\
\hline Largest diff. peak and hole [e $\AA^{-3}$ ] & $0.234 /-0.382$ & $0.334 /-0.338$ \\
\hline
\end{tabular}

Table 1. Parameters of the single crystals, data collection and structure refinement of $\mathbf{3}$ and $\mathbf{7}$.

coordination to the $\operatorname{Ln}(\mathrm{III})$ ions. The complexes were purified on an Agilent-Prep-C18 column (solvent A: $0.1 \%$ TFA in water; solvent B: $10 \%$ of $0.1 \%$ TFA, $90 \%$ aq. $\left.\mathrm{CH}_{3} \mathrm{CN}\right)$. Yield $\sim 30 \mathrm{mg}$. - MALDI-TOF MS: $\mathrm{m} / z=712$ $\left(\mathrm{C}_{27} \mathrm{H}_{40} \mathrm{~N}_{7} \mathrm{O}_{10} \mathrm{Y},[\mathrm{M}+\mathrm{H}]^{+}\right) ; m / z=788\left(\mathrm{C}_{27} \mathrm{H}_{40} \mathrm{~N}_{7} \mathrm{O}_{10} \mathrm{Ho}\right.$, $\left.[\mathrm{M}+\mathrm{H}]^{+}\right) ; m / z=792\left(\mathrm{C}_{27} \mathrm{H}_{40} \mathrm{~N}_{7} \mathrm{O}_{10} \mathrm{Tm},[\mathrm{M}+\mathrm{H}]^{+}\right) ; \mathrm{m} / z=$ $798\left(\mathrm{C}_{27} \mathrm{H}_{40} \mathrm{~N}_{7} \mathrm{O}_{10} \mathrm{Lu},[\mathrm{M}+\mathrm{H}]^{+}\right)$. The analytical HPLC method for the metal-DOTA-conjugates used an Agilent 1100 HPLC system and was performed on a Zorbax 300SB-C18 $4.6 \times 150 \mathrm{~mm}$ column (Agilent) with a flow rate of $1 \mathrm{~mL} / \mathrm{min}$ and a linear gradient of $100 \%$ solution $\mathrm{A}$ to $60 \%$ solution $\mathrm{B}$ in 30 min (A: $0.1 \%$ TFA in water; solvent B: $0.1 \%$ TFA, $90 \%$ aq. $\left.\mathrm{CH}_{3} \mathrm{CN}\right)$ with spectrophotometric monitoring at $\lambda=220 \mathrm{~nm}$. The retention time was the same $(9.05 \mathrm{~min})$ for all complexes. 18, 19, 20 and 21: decomposition $>220{ }^{\circ} \mathrm{C}$. 21: ${ }^{1} \mathrm{H}$ NMR $(400 \mathrm{MHz}$, $\left.\mathrm{D}_{2} \mathrm{O}\right): \delta=1.40\left(\mathrm{~m}, 2 \mathrm{H}, \mathrm{NHCH}_{2} \mathrm{CH}_{2} \mathrm{CH}_{2}\right), 1.45(\mathrm{~m}, 2 \mathrm{H}$, $\mathrm{NHCH}_{2} \mathrm{CH}_{2} \mathrm{CH}_{2}$ ), 2.23-2.82 (broad, $12 \mathrm{H}, \mathrm{NCH}_{2} \mathrm{CH}_{2} \mathrm{~N}$; $2 \mathrm{H}, \mathrm{NCH}_{2} \mathrm{CH}_{2} \mathrm{CO}$ ), 3.09 (t, $2 \mathrm{H}, \mathrm{CONHCH}_{2}$ ), 3.12 (m, $2 \mathrm{H}, \mathrm{CONHCH}_{2}$ ), $3.12-3.73$ (broad, $8 \mathrm{H}, \mathrm{NHCH}_{2} \mathrm{CO}$; broad $4 \mathrm{H}, \mathrm{NCH}_{2} \mathrm{CH}_{2} \mathrm{~N}$ ), 3.75 (broad, $2 \mathrm{H}, \mathrm{NCH}_{2} \mathrm{CH}_{2} \mathrm{CO}$ ), $6.74(\mathrm{~s}, 2 \mathrm{H}, \mathrm{CH}=\mathrm{CH}) .-{ }^{13} \mathrm{C} \mathrm{NMR}\left(100.64 \mathrm{MHz}, \mathrm{D}_{2} \mathrm{O}\right)$ : $\delta=26.6\left(\mathrm{NHCH}_{2} \mathrm{CH}_{2} \mathrm{CH}_{2}\right), 26.7\left(\mathrm{NHCH}_{2} \mathrm{CH}_{2} \mathrm{CH}_{2}\right), 34.3$ $\left(\mathrm{NCH}_{2} \mathrm{CH}_{2} \mathrm{CO}\right), 38.2\left(\mathrm{NCH}_{2} \mathrm{CH}_{2} \mathrm{CO}\right), 39.8\left(\mathrm{CH}_{2} \mathrm{NHCO}\right)$, $40.0\left(\mathrm{CONHCH}_{2}\right), 54.3-56.8\left(8 \times \mathrm{CH}_{2}, \mathrm{NCH}_{2} \mathrm{CH}_{2} \mathrm{~N}\right), 63.4$ $\left(1 \times \mathrm{CH}_{2}, \mathrm{NHCH}_{2} \mathrm{CO}\right), 65.7\left(3 \times \mathrm{CH}_{2}, \mathrm{NHCH}_{2} \mathrm{CO}\right), 134.8$ $(\mathrm{CH}=\mathrm{CH}), 171.4\left(\mathrm{NCH}_{2} \mathrm{CH}_{2} \mathrm{CO}\right), 174.2(=\mathrm{CHCO}), 180.9$
$(1 \times \mathrm{CO}, C \mathrm{O}), 181.2(3 \times \mathrm{CO}, C \mathrm{O}) .-\mathrm{C}_{27} \mathrm{H}_{40} \mathrm{~N}_{7} \mathrm{O}_{10} \mathrm{Lu}$ (797.65): calcd. C 40.66, H 5.05, N 12.29; found C 40.39, H 5.12, N 12.12 .

\section{Crystal structure determination}

Crystals suitable for X-ray diffraction were obtained by crystallization of $\mathbf{3}$ from the pure oil and of $\mathbf{7}$ from acetone. The data were collected on a Siemens SMART CCD diffractometer (graphite monochromated $\mathrm{Mo} K_{\alpha}$ radiation, $\lambda=0.71070 \AA$ ) by use of $\omega$ scans at $293 \mathrm{~K}$ (3) and $173 \mathrm{~K}(7)$. The structures were solved by Direct Methods using SHELXS-97 [35] and refined on $F^{2}$ using all reflections with SHELXL-97 [36]. All non-hydrogen atoms were refined anisotropically and the carbon-bound hydrogen atoms were placed in calculated positions and assigned to an isotropic displacement parameter of $U_{\text {iso }}=$ $0.08 \AA^{2}$. Hydrogen atoms bonded to nitrogen were found. SADABS [37] was used to perform area-detector scaling and absorption corrections. Important parameters of the single crystals, data collection and the refinement of the structure are listed in Table 1. Further crystallographic data were deposited as supplementary publication no. CCDC 608991 (7) und CCDC 608992 (3) and can be obtained free of charge from The Cambridge Crystallographic Data Centre via www.ccdc.cam.ac.uk/data_request/cif.

Acknowledgements 
This work was supported by the Deutsche Forschungsgemeinschaft (SPP "Lanthanoidspezifische Funktionalitäten in Molekül und Material") and the Fonds der Chemischen Industrie. We thank Barbara Brecht-Jachan, Prisca Kunert and
Dr. Peter Henklein, Universitätsklinikum Charité, HumboldtUniversität $\mathrm{zu}$ Berlin, for the purification of compounds 16- 21.
[1] S. Liu, D. S. Edwards, Bioconjugate Chem. 2001, $12,7$.

[2] V. Jacques, J. Desreux in The Chemistry of Contrast Agents in Medical Magnetic Resonance Imaging (Eds.: A. E. Merbach, E. Tóth), Wiley, Chichester, 2001, p. 157.

[3] P. Caravan, J. J. Ellison, T. J. McMurry, R. B. Lauffer, Chem. Rev. 1999, 99, 2293.

[4] R. R. Edelman, J.R. Hesselink, M. B. Zlatkin in MRI: Clinical Magnetic Resonance Imaging, Saunders, Philadelphia, 1996.

[5] D. C. Onthank, S. Liu, P. J. Silva, J. A. Barrett, T. D. Harris, S.P. Robinson, D. S. Edwards, Bioconjugate Chem. 2004, 15, 235.

[6] M. Woods, A. D. Sherry, Inorg. Chem. 2003, 42, 4401.

[7] M. Krause, C. Scheler, U. Boettger, H. Weisshoff, M. Linscheid, DE10227599A1 (Proteome Factory AG, Humboldt University Berlin) 2002.

[8] P. A. Whetstone, N. G. Butlin, T. M. Corneillie, C.F. Meares, Bioconjugate Chem. 2004, 15, 3.

[9] A. J. Link, Electrophoresis 1997, 18, 1314.

[10] A. Shevchenko, Proc. Natl. Acad. Sci. USA 1996, 93, 1440.

[11] S. P. Gygi, B. Rist, T. J. Griffin, J. Eng, R. Aebersold, J. Proteome Res. 2002, 1, 47.

[12] R. Aebersold, M. Mann, Nature 2003, 422, 198.

[13] S. P. Gygi, B. Rist, S. A. Gerber, F. Turecek, M. H. Gelb, R. Aebersold, Nat. Biotechnol. 1999, 17, 994.

[14] T. M. Corneillie, A. J. Fisher, C.F. Meares, J. Am. Chem. Soc. 2003, 125, 15039.

[15] J. P. L. Cox, A. S. Craig, I. M. Helps, K. J. Jankowski, D. Parker, M. A. W. Eaton, A. T. Millican, K. Millar, N. R. A. Beeley, B.A. Boyce, J. Chem. Soc., Perkin Trans. I 1990, 2567.

[16] D. D. Dischino, E. J. Delaney, J.E. Emswiler, G. T. Gaughan, J. S. Prasad, S. K. Srivastava, M. F. Tweedle, Inorg. Chem. 1991, 30, 1265.

[17] A. Heppeler, S. Froidevaux, H. R. Mäcke, E. Jermann, M. Béhé, P. Powell, M. Hennig, Chem. Eur. J. 1999, 5, 1974.

[18] N. V. Gerbeleu, V. B. Arion, J. Burgess, Template Synthesis of Macrocyclic Compounds, Wiley-VCH, Weinheim, 1999.

[19] H. Bernard, J. J. Yaouanc, J.C. Clement, H. des Abbayes, H. Handel, Tetrahedron Lett. 1991, 32, 639.
[20] J.P. André, C. F. G. C. Geraldes, J. A. Martins, A. E. Merbach, M. I. M. Prata, A. C. Santos, J. J.P. de Lima, E. Tóth, Chem. Eur. J. 2004, 10, 5804.

[21] H. Schumann, K. Kuse, S. Dechert, Z. Naturforsch. 2004, 59b, 1415.

[22] A.E. Martin, T.M. Ford, J.E. Bulkowski, J. Org. Chem. 1982, 47, 412.

[23] T. J. Atkins, J. E. Richman, W. F. Oettle, Org. Synth. 1978, $58,86$.

[24] F. P. Schmidtchen, Chem. Ber. 1980, 113, 2175.

[25] J.E. Richman, T. J. Atkins, J. Am. Chem. Soc. 1974, 96, 2268.

[26] D. Parker, Macrocycle Synthesis. A Practical Approach. Oxford University Press, 1996.

[27] V. Patinec, J. J. Yaouanc, J.C. Clément, H. Handel, H. des Abbayes, M. M. Kubicki, J. Organomet. Chem. 1995, 494, 215.

[28] V. Patinec, I. Gardinier, J. J. Yaouanc, J. C. Clément, H. Handel, H. des Abbayes, Inorg. Chim. Acta 1996, 244, 105.

[29] Diamond, Crystal and Molecular Structure Visualization, Crystal Impact - K. Brandenburg \& H. Putz GbR, Bonn (Germany) 2004.

[30] M. S. Ali, S. M. Quadri, Bioconjugate Chem. 1996, 7, 576.

[31] L.A. Carpino, H. Imazumi, A. El-Faham, F. J. Ferrer, C. Zhang, Y. Lee, B. M. Foxman, P. Henklein, C. Hanay, C. Mügge, H. Wenschuh, J. Klose, M. Beyermann, M. Bienert, Angew. Chem. 2002, 114, 458.

[32] C. Li, W. T. Wong, J. Org. Chem. 2003, 68, 2956.

[33] M. P. Hall, S. Ashrafi, I. Obegi, R. Petesch, J. N. Peterson, L. V. Schneider, J. Mass. Spectrom. 2003, 38, 809.

[34] C.F.G.C. Geraldes, A. D. Sherry, M. P. M. Marques, M. C. Alpoim, S. Cortes, J. Chem. Soc., Perkin Trans. 1991, 137.

[35] G. M. Sheldrick, SHELXS-97, Program for the Solution of Crystal Structures, University of Göttingen, Göttingen (Germany) 1990.

[36] G. M. Sheldrick, SHELXL-97, Program for the Refinement of Crystal Structures, University of Göttingen, Göttingen (Germany) 1997.

[37] G. M. Sheldrick, SADABS, Program for Empirical Absorption Correction of Area Detector Data, University of Göttingen, Göttingen (Germany) 1996. 